\title{
Development and Characterization of Lipid-Based Nanosystems: Effect of Interfacial Composition on Nanoemulsion Behavior
}

\author{
Hélder D. Silva ${ }^{1} \cdot$ Miguel A. Cerqueira ${ }^{1,2}$ (1) - Francesco Donsì ${ }^{3}$ - Ana C. Pinheiro ${ }^{1} \cdot$ Giovanna Ferrari $^{3,4}$ $^{-}$ \\ António A. Vicente ${ }^{1}$
}

Received: 21 March 2019 / Accepted: 29 October 2019 / Published online: 26 November 2019

(C) Springer Science+Business Media, LLC, part of Springer Nature 2019

\begin{abstract}
Nanoemulsions were successfully developed through high-pressure homogenization. The layer-by-layer electrostatic technique was used for the subsequent deposition of a chitosan and alginate polyelectrolyte layers, thus leading to the development of a multilayer nanoemulsion. The effect of polyelectrolytes concentration in the development of multilayer nanoemulsions was evaluated in terms of hydrodynamic diameter $\left(H_{d}\right)$, polydispersity index $(P d I)$, zeta potential $(Z p)$, and curcumin encapsulation efficiency. The interactions between polyelectrolytes and nanoemulsion were further analyzed using Fourier transform infrared (FTIR) spectroscopy and quartz crystal microbalance (QCM), while curcumin degradation was determined through the evaluation of the antioxidant capacity of the nanosystems. Results showed an encapsulation efficiency of $99.8 \pm 0.8 \%$ and a loading capacity of $0.53 \pm 0.03 \%(\mathrm{w} / \mathrm{w})$. The presence of the multilayers leads to an increase of the $H_{d}$ of the nanosystems, from $80.0 \pm$ $0.9 \mathrm{~nm}$ (nanoemulsion) to $130.1 \pm 1.5 \mathrm{~nm}$ (multilayer nanoemulsion). Release profiles were evaluated at different conditions, fitting a linear superposition model to experimental data suggests an anomalous behavior, being the relaxation of the surfactant and polyelectrolytes the rate-determining phenomena in curcumin release. The developed nanosystems showed great potential for the incorporation of lipophilic bioactive compounds, in view of their application in food and pharmaceutical products.
\end{abstract}

Keywords Multilayer $\cdot$ Nanoemulsion $\cdot \mathrm{pH}$-responsive behavior $\cdot$ Curcumin degradation $\cdot$ Controlled release

\section{Introduction}

In recent years, one of the focuses of the food industry is the lipophilic bioactive compounds. Their health-promoting properties and encapsulation-related issues, such as low solubility in aqueous mediums and low bioaccessibility, led to several studies on this field (McClements \& Xiao, 2012; Pinheiro, Coimbra, \& Vicente 2016). Curcumin [1,7-bis(4-hydroxy-3-

Miguel A. Cerqueira

miguel.cerqueira@inl.int

1 Centre of Biological Engineering, University of Minho, 4710-057 Braga, Portugal

2 International Iberian Nanotechnology Laboratory, Av. Mestre José Veiga s/n, 4715-330 Braga, Portugal

3 Department of Industrial Engineering, University of Salerno, Fisciano, Italy

4 ProdAl Scarl, Competence Center on Agro-Food Productions, University of Salerno, Fisciano, Italy methoxyphenyl)-1,6-hepadiene-3,5-dione], a natural polyphenol, extracted from the rhizome of Curcuma longa, has been reported to have a wide range of biological activities, such as antioxidant, anti-inflammatory, antimicrobial, and anticancer properties (Kaur, Kumar, \& Mehta 2015; Liu, Cai, Jiang, Wu, \& Le 2016). Nonetheless, the application of curcumin to food products has been limited due to their low solubility in water $(11 \mathrm{ng} / \mathrm{mL})$, rapid degradation in aqueous systems due to oxidative processes, $\mathrm{pH}$ and temperature changes during manufacturing or storage of the food products, and their effect on the organoleptic properties of foods (Plaza-Oliver et al. 2015; Zhao et al. 2012). The need to overcome these limitations (from curcumin and other lipophilic compounds) has made that the food and beverage industry considered the use of encapsulation systems at the nanoscale. Among encapsulation systems, cyclodextrins (Li, Zhang, Liu, Guo, \& Qiao 2018; Tomren, Másson, Loftsson, \& Tønnesen 2007), zein nanocapsules (Hu et al. 2015), and lipid-based nanosystems (Silva, Cerqueira, \& Vicente 2012) have been used for this purpose. Lipid-based nanosystems are able to encapsulate lipophilic bioactive compounds efficiently, helping their 
incorporation into food products while protecting the bioactive compounds and acting as a delivery system (Ezhilarasi, Karthik, Chhanwal, \& Anandharamakrishnan, 2013; Spigno et al. 2013; Cerqueira et al. 2014; Plaza-Oliver et al. 2015; Pinheiro et al. 2016).

The use of lipid-based nanosystems, such as nanoemulsions, is an interesting option for the food and beverage industries to encapsulate lipophilic bioactive compounds, protecting their unique properties, while releasing them at the desired target. Nanoemulsions exhibit several distinctive characteristics, such as high optical clarity, good physical stability, and protection of the incorporated bioactive compounds from degradation, while also improving their bioavailability (Silva, Silva et al. 2012; Guttoff, Saberi, \& McClements 2015; Saberi, Zeeb, Weiss, \& McClements 2015; Silva, Cerqueira, \& Vicente 2015b). These characteristics can help preserve the organoleptic and nutritional properties of food products when lipophilic bioactive compounds are incorporated (Plaza-Oliver et al. 2015). Nevertheless, nanoemulsions present some drawbacks such as limited stability to heating, chilling, freezing-thawing, and dehydration (Cerqueira et al. 2014; Silva, Cerqueira, \& Vicente 2015a). Previous studies have shown that the surface properties of nanoemulsions can be modified through the addition of a polyelectrolyte layer (Li et al. 2010; Choi, Kim, Cho, Hwang, \& Kim 2011; Saberi et al. 2015; Pinheiro et al. 2016); however, its behavior under different environmental stresses and food systems is not fully understood. This deposition can be performed through the layer-by-layer (LbL) electrostatic deposition technique, a powerful tool for the alternate deposition of polyelectrolytes and thus the development of multilayer nanoemulsions with improved stability against environmental stresses (Cerqueira et al. 2014; Li et al. 2010; Pinheiro et al. 2016). LbL deposition changes the surface properties of nanoemulsions and creates the possibility of tailoring the properties of lipid-based systems, offers a precise control over thickness and morphology, while allows the controlled release of the lipophilic bioactive compounds (Cui, van Koeverden, Müllner, Kempe, \& Caruso 2014). LbL electrostatic deposition technique relies on the electrostatic interactions between a charged polyelectrolyte deposited on the surface of an oppositely charged system. However, the selection of the composition of those systems must be carefully controlled in order to avoid particle aggregation due to bridging or depletion flocculation (Choi et al. 2011; Cui et al. 2014; Saberi et al. 2015). Therefore, it is important to fully understand the effect of polyelectrolyte layers in the stability of nanoemulsions and how it will influence the release behavior of bioactive compounds. To the best of the authors' knowledge, this kind of studies (with multilayer nanoemulsions) is lacking and only a few and very recent studies can be found ( $\mathrm{Li}$ et al. 2019), despite the several studies evaluating the release of bioactive compounds from simple nanoemulsions in different medium (Le et al. 2011; Qian et al. 2012; Artiga-Artigas et al. 2018). Li et al. (2019) evaluated the spontaneously ordered adsorption of different materials (whey protein hydrolysate, sodium caseinate, and gum Arabic) to stabilize the nanoemulsions and do not use the LbL electrostatic deposition technique approach as presented in this work.

The objective of this work was to evaluate the effect the multilayers on the stability and release of curcumin from nanoemulsions. Therefore, multilayer curcumin-loaded nanoemulsions were developed by the addition of polyelectrolytes (alternate deposition of chitosan and alginate layers) using the LbL deposition technique. They were then characterized in terms of size, polydispersity, and zeta potential and the release of curcumin from nanoemulsions was evaluated in different media. In order to understand the behavior of the nanoemulsions under environmental stresses, nanoemulsions' stability was evaluated under different temperatures and $\mathrm{pH}$ and during storage.

\section{Materials and Methods}

\section{Materials}

Neobee 1053 medium chain triglycerides (MCTs) is a caprylic/capric triglyceride oil with a fatty acid distribution of $55 \%$ of $\mathrm{C} 8: 0$ and $44 \%$ of $\mathrm{C} 10: 0$ which was kindly provided by Stepan (The Netherlands) and used without further purification. Sodium dodecyl sulphate (SDS), curcumin $(\mathrm{Mw}=$ 368.38 Da), and 1,1-diphenyl-2-picrylhydrazyl (DPPH) were purchased from Sigma-Aldrich (St Louis, MO, USA). Chitosan (deacetylation degree $\geq 95 \%$ ) with $\mathrm{Mw} \approx$ 300,000 Da was purchased from Golden-Shell Biochemical CO., LTD (Zhejiang, China) and sodium alginate from Manutex RSX, Kelco 104 International, Ltd. (Portugal) with $\mathrm{Mw} \approx 15,900 \mathrm{Da}$ and viscosity $\approx 200 \mathrm{cp}(1 \%$ aqueous solution with Brookfield Model LV $-60 \mathrm{rpm}$ at $25^{\circ} \mathrm{C}$ ). Lactic acid (90\%) was obtained from Acros Organics (Geel, Belgium) and ethanol was obtained from Panreac (Spain). Distilled water (Milli-Q apparatus, Millipore Corp., Bedford, MA, USA) was used to prepare all solutions.

\section{Experimental Procedures}

\section{Preparation of Curcumin Nanosystems}

Curcumin Nanoemulsion Preparation Oil-in-water $(\mathrm{O} / \mathrm{W})$ nanoemulsions were prepared according to Silva et al. (2015b), with minor modifications. Briefly, $0.1 \%$ (w/w) of curcumin was solubilized at $90{ }^{\circ} \mathrm{C}$ in MCTs (Yu, Shi, Liu, \& Huang 2012; Yucel, Quagliariello, Iaffaioli, Ferrari, \& Donsì 2015). This oily phase was then added to an aqueous phase containing $1 \%(\mathrm{w} / \mathrm{w})$ of SDS in distilled water. According to previous works (Silva et al. 2011; Silva et al. 2015b), an oily 
phase and aqueous phase volume ratio of 1:9 was used. The emulsions were pre-mixed during $2 \mathrm{~min}$ at $5000 \mathrm{rpm}$ using an Ultra-Turrax homogenizer (T 25, Ika-Werke, Germany) followed by 20 passages through a high-pressure homogenizer equipped with a zirconia nozzle (Z4 nozzle) with $100 \mu \mathrm{m}$ of orifice (Nano DeBEE, BEE International, USA) at 15000 Psi. The surfactant used in this study was applied as a model surfactant based on its negative charge, since one of the aims of this study was to produce multilayer nanoemulsions using the LbL electrostatic deposition technique. SDS can be used in the EUA (and other countries that closely follow FDA's regulations) in food products, e.g., as surfactant in fruit juice drinks below $25 \mathrm{ppm}$ and in coatings on fresh citrus fruits (Food and Drug Administration 2019). However, it is not allowed in Europe according to the European Food Safety Authority (EFSA) (EFSA, 2010).

Curcumin Multilayer Nanoemulsion Preparation The multilayer nanoemulsions were formed through adsorption of consecutive deposition of layers of polyelectrolytes onto the curcumin nanoemulsions using the LbL electrostatic deposition technique. The saturation method was used to determine the optimum chitosan and sodium alginate concentrations required to prepare the multilayer nanoemulsions, i.e., the layers were constructed by subsequent adsorption of polyelectrolytes from their solutions without the intermediate rinsing step (Adamczak, Kupiec, Jarek, Szczepanowicz, \& Warszyński 2014). Briefly, anionic curcumin nanoemulsions were coated with alternating layers of positively charged chitosan solution and negatively charged sodium alginate (volume ratio of 1:1, respectively) until the desired number of three polyelectrolyte layers was achieved (nanoemulsion-chitosan-alginate-chitosan). The polyelectrolyte solutions were added dropwise with a syringe pump (NE-1000, New Era Pump Systems, Inc., USA) to fresh curcumin nanoemulsions, under stirring for $15 \mathrm{~min}$. The $\mathrm{pH}$ of the nanoemulsion after production was 6.7; nevertheless, in order to promote the construction of the multilayers, the $\mathrm{pH}$ of the nanoemulsions and multilayer nanoemulsions was maintained at 5.0 using solutions of $0.1 \mathrm{M} \mathrm{NaOH}$ or $0.1 \mathrm{M} \mathrm{HCl}$. The concentrations of polyelectrolyte solutions used to form each layer were selected on the basis of the results of zeta potential $(Z p)$ measurements (Adamczak et al. 2014; Madrigal-Carballo et al. 2010).

\section{Size Measurements}

The particle size distribution and the polydispersity index $(P d I)$ of nanoemulsions and multilayer nanoemulsions were determined using dynamic light scattering (DLS) (Zetasizer Nano ZS-90, Malvern Instruments, Worcestershire, UK). The nanoemulsions were diluted $100 \times$ in distilled water at room temperature, without affecting $H_{d}$ and $P d I$. Data were reported as the mean droplet diameter (hydrodynamic diameter, $H_{d}$ ). $P d I$ is a dimensionless number and indicates the heterogeneity (monodisperse or polydisperse) of sizes of particles in a mixture (Malvern, 2011). Each sample was analyzed in a disposable polystyrene cell (DTS0012, Malvern Instruments, Worcestershire, UK). The measurements were performed in duplicate, with three readings for each of them. The results are given as the average \pm standard deviation of the six values obtained (Rao \& McClements, 2013; Silva et al. 2011).

The theoretical size of the nanoemulsions produced by high-pressure homogenization at $1 \%(\mathrm{w} / \mathrm{w})$ of surfactant can be calculated using Eq. 1.

$R_{\text {min }}=\frac{3 * \Gamma * \phi}{c_{S}^{\prime} *(1-\phi)}$

Here, $\Gamma$ is the surface load of the surfactant at saturation $\left(\mathrm{kg} / \mathrm{m}^{2}\right), \phi$ is the disperse phase volume (dimensionless), and $C_{s}^{\prime}$ is the concentration of surfactant at the continuous phase $\left(\mathrm{kg} / \mathrm{m}^{3}\right)$ (Troncoso, Aguilera, \& McClements 2012).

\section{Charge Measurements}

The droplet charge $(Z p)$ of the nanoemulsions and multilayer nanoemulsions was determined using a particle microelectrophoresis instrument (Zetasizer Nano ZS-90, Malvern Instruments, Worcestershire, UK) and the Smoluchowski approximation. Nanoemulsions were diluted $100 \times$ in distilled water prior to measurements in order to avoid multiple scattering effects at ambient temperature and placed into disposable capillary cells (DTS1060, Malvern Instruments, Worcestershire, UK) (Ozturk, Argin, Ozilgen, \& McClements 2014; Rao \& McClements 2013).

\section{Evaluation of Temperature and $\mathrm{pH}$ Responses}

Thermal Stress The influence of temperature on nanoemulsions stability was examined through dynamic light scattering (DLS), by measuring the $H_{d}$ of the nanoemulsions. Briefly, $1 \mathrm{~mL}$ of the formulations was submitted to a range of temperatures from 10 to $80^{\circ} \mathrm{C}$, with increasing intervals of 10 ${ }^{\circ} \mathrm{C}$, during $10 \mathrm{~min}$ at each condition (Morais Diane \& Burgess, 2014). The temperature was adjusted using a Nano ZS-90 equipment (Zetasizer Nano ZS-90, Malvern Instruments, Worcestershire, UK). The occurrence of creaming was visually checked during experiment and excluded if it was the case. Data were reported as the mean droplet diameter (hydrodynamic diameter, $\left.H_{d}\right)$, mean $\pm \operatorname{SD}(n=3)$.

pH Stress The $\mathrm{pH}$-responsive behavior of the nanosystems was analyzed by measuring the droplets mean diameter to responses to dynamic $\mathrm{pH}$ values change using DLS, Zetasizer Nano ZS operating with a 633-nm laser source equipped with a MPT-2 Autotitrator. The $\mathrm{pH}$ value of the 
nanosystems was adjusted to 2 and gradually increased to 12 using 0.1 and $0.5 \mathrm{~mol} \mathrm{~L}^{-1} \mathrm{NaOH}$ solution and $0.1 \mathrm{~mol} \mathrm{~L}^{-1}$ $\mathrm{HCl}$ solution. Measurements of the $H_{d}$ were collected at $25^{\circ} \mathrm{C}$ and $\mathrm{pH}$ intervals of 1 (Liechty, Scheuerle, \& Peppas 2013).

\section{Encapsulation Efficiency and Loading Capacity of Curcumin Nanoemulsions}

The encapsulation efficiency (EE) and loading capacity (LC) were determined by measuring the amount of curcumin that was not encapsulated within the nanoemulsions. Briefly, 0.5 $\mathrm{mL}$ of the curcumin nanoemulsion solution was placed into an Amicon ${ }^{\circledR}$ Ultra 0.5 centrifugal filter with $10 \mathrm{kDa}$ cutoff (Amicon ${ }^{\circledR}$ Ultra $-0.5 \mathrm{~mL}$ device, Millipore Corp., Ireland) and centrifuged at $14,000 \mathrm{~g}$ for $15 \mathrm{~min}$. Efficient recovery of the concentrated sample (retained species) is achieved by a reverse spin step at 14,000 $\mathrm{g}$ after collecting the filtrate. This allowed the separation of the unabsorbed curcumin (filtered) from the nanoemulsions with encapsulated curcumin (supernatant). In order to measure the EE (Eq. 2), the free curcumin in the filtrate was assayed spectrophotometrically at $425 \mathrm{~nm}$, the maximum absorbance peak of curcumin, in Elisa Biotech Synergy HT (Biotek, USA). This procedure was performed twice, being the unabsorbed curcumin removed by two repeated cycles of centrifugation. For LC determination (Eq. 3), the filtered nanoemulsions were dried and weighted.

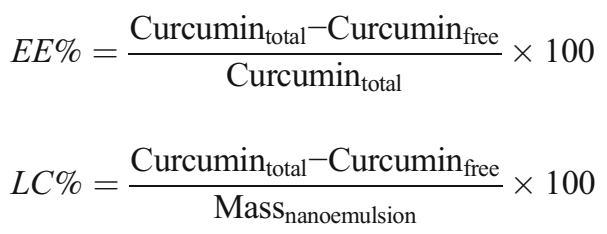

Curcumin $_{\text {total }}$ represents the total amount of curcumin added to the system, curcumin $_{\text {free }}$ is the free/unabsorbed

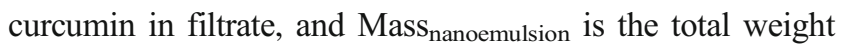
of nanoemulsions after drying (Azevedo, Bourbon, Vicente, \& Cerqueira 2014).

\section{Antioxidant Activity of Curcumin Nanosystems}

The free-radical scavenging capacity of curcumin and curcumin nanosystems were analyzed using the 1,1diphenyl-2-picrylhydrazyl (DPPH) test according to the methodology described by Pinheiro et al. (2015), with some modifications. Briefly, $0.2 \mathrm{~mL}$ of ethanol and $0.3 \mathrm{~mL}$ of the curcumin dissolved in ethanol (concentrations ranging from 0.05 to $5.0 \mathrm{mg} \mathrm{mL}^{-1}$ ) was mixed in a $10-\mathrm{mL}$ test tube with 2.5 $\mathrm{mL}$ of DPPH (60 $\mu \mathrm{mol} \mathrm{L}^{-1}$ in ethanol), achieving a final volume of $3.0 \mathrm{~mL}$. The solution was kept at room temperature for $30 \mathrm{~min}$ and the absorbance was measured at $517 \mathrm{~nm}$ (Pinheiro et al. 2015; Rufino et al. 2007; Souza et al. 2012).
Butylated hydroxytoluene (BHT) and butylated hydroxyanisole (BHA) were used as positive controls. Also, blank nanoemulsions were used as a negative control.

The DPPH scavenging effect was calculated as follows:

Scavenging effect $(\%)=\frac{A_{0}-\left(A_{s}-A_{b}\right)}{A_{0}} \times 100$

where $A_{0}$ is the absorbance at $517 \mathrm{~nm}$ of DPPH without sample, $A_{s}$ is the absorbance at $517 \mathrm{~nm}$ of sample and DPPH, and $A_{b}$ is the absorbance at $517 \mathrm{~nm}$ of sample without DPPH. The absorbance measurements were performed in Elisa Biotech Synergy HT (Biotek, USA) (Pinheiro et al. 2015).

\section{Fourier Transform Infrared Spectroscopy}

In order to confirm the successful development of the multilayer nanoemulsions, Fourier transform infrared (FTIR) analyses were carried out with a Thermo Nicolet 6700 spectrometer (Thermo Scientific, Waltham, MA, USA) in the wavenumber region of $600-4000 \mathrm{~cm}^{-1}$ using 16 scans for each sample. The nanosystems were air dried prior to FTIR measurements to avoid interferences from water. Each spectrum was baseline corrected and the transmittance was normalized between 0 and 1 .

\section{Quartz Crystal Microbalance}

The adsorption of the polyelectrolytes onto the curcumin nanoemulsions was evaluated using a quartz crystal microbalance (QCM 200, purchased from Stanford Research Systems, SRS, USA), equipped with AT-cut quartz crystals (5 MHz) with optically flat polished chrome/gold electrodes on contact and liquid sides. Before carrying out the experiments, the crystal was cleaned by successive sonication $(40 \mathrm{kHz}, 30$ $\mathrm{min}$ ) in ultra-pure water, ethanol, and ultra-pure water, followed by drying with a flow of nitrogen. Adsorption measurements were performed by alternate immersion of the crystal in nanoemulsions ( $\mathrm{pH}$ 5.0), chitosan ( $\mathrm{pH} 3.0$ ), alginate ( $\mathrm{pH} 7.0)$, and chitosan (pH 3.0) solutions, for $15 \mathrm{~min}$. The variations of the resonance frequency $(F)$ were simultaneously measured as a function of time and the analyses were performed at $25^{\circ} \mathrm{C}$, in triplicate (Pinheiro et al. 2015).

\section{Microscopy}

The morphology of nanosystems was evaluated by transmission electron microscopy (TEM) (EM 902A, ZEISS, Germany) operating at $80 \mathrm{kV}$. TEM samples were prepared by depositing the nanoemulsion suspensions on a carbon-coated copper grid, and negatively stained with $1 \%(\mathrm{w} / \mathrm{v})$ uranyl acetate for observation. Samples were air-dried before analyses. 


\section{Curcumin Release from Nanosystems}

The in vitro release kinetics of curcumin was performed by the dialysis method (Azevedo et al. 2014; Pinheiro et al. 2015). Two milliliters of aqueous curcumin nanosystems was added into a dialysis membrane (molecular weight cutoff $15 \mathrm{kDa}$; Cellu-Sep H1, Membrane filtration products, USA). The sealed dialysis membrane was then placed into $50 \mathrm{~mL}$ of acceptor medium $(10,20$, and $50 \%$ $\mathrm{v} / \mathrm{v}$ of ethanol and $5 \% \mathrm{w} / \mathrm{w}$ of SDS) under magnetic stirring at $4{ }^{\circ} \mathrm{C}, 25^{\circ} \mathrm{C}$, and $37{ }^{\circ} \mathrm{C}$. At appropriate time intervals, $0.5 \mathrm{~mL}$ of supernatant was taken and $0.5 \mathrm{~mL}$ of fresh acceptor medium was added to keep the volume of the release medium constant. The released amount of curcumin from the nanosystems was evaluated by measuring the absorbance at $425 \mathrm{~nm}$, maximum absorbance peak (Elisa Biotech Synergy HT, Biotek, USA). All release tests were run in duplicate or triplicate. In order to evaluate the release mechanism of curcumin from nanosystems, the linear superposition model (LSM) was applied (Eq. 5). This kinetic model accounts for both Fickian and Case II transport effects (Berens \& Hopfenberg, 1978).

$M_{t}=M_{t, F}+M_{t, R}$

where $M_{t}$ is the total amount of sorption per unit weight of nanosystems at time $t$, being $M_{t, F}$ and $M_{t R}$ are the Fickian and polymer relaxation contributions, respectively, at time $t$. Pure Fickian diffusion can be described by Eq. 6 :

$M_{t, F}=M_{\infty, F}\left[1-\frac{6}{\pi^{2}} \sum_{n=1}^{\infty} \frac{1}{n^{2}} \exp \left(-n^{2} k_{F} t\right)\right] M_{t, R}$

where $M_{\infty, F}$ is the compound release at equilibrium and $k_{F}$ is the Fickian diffusion rate constant. Eq. 6 can be simplified using the first term of the Taylor series (Pinheiro et al. 2015; Pinheiro, Bourbon, Quintas, Coimbra, \& Vicente 2012).

Polymer relaxation (Eq. 7) is driven by the swelling ability of the polymer being then related to the dissipation of stress induced by the entry of the penetrant and can be described as a distribution of relaxation times, each assuming a first ordertype kinetic equation (Berens \& Hopfenberg 1978).

$M_{t, R}=\sum_{i} M_{\infty, R_{i}}\left[1-\exp \left(-k_{R_{i}} t\right)\right]$

where $M_{\infty, R i}$ are the contributions of the relaxation processes for compound release and $k_{R i}$ are the relaxation rate constants. For most cases, there is only one main polymer relaxation that influences transport and thus the above equation can be simplified using $i=1$. Therefore, Eq. 8 can describe the linear superposition model for curcumin release from nanosystems.
$\frac{M_{t}}{M_{\infty}}=M_{F}\left[1-\frac{6}{\pi^{2}} \exp \left(k_{F} t\right)\right]+M_{R}\left[1-\exp \left(k_{R} t\right)\right]$

where $M_{F}$ is the total mass of compound released by Fickian transport and $M_{R}$ is the total mass of compound released by polymer relaxation. The experimental data were analyzed by fitting Eq. 8 (linear superposition model) in order to assess the transport mechanism involved in the curcumin release from nanosystems at different temperatures and acceptor media (Pinheiro et al. 2015).

\section{Stability of the Nanosystems Under Storage}

In order to evaluate the stability of nanoemulsion and multilayer nanoemulsion during storage, the $H_{d}, P d I$, and $Z p$ were evaluated during three months of storage at $4{ }^{\circ} \mathrm{C}$ in the absence of light.

\section{Statistical Procedures}

\section{Data Analyses}

Data analyses were performed using Microsoft Windows Excel 2011, using Tukey's Multiple Comparison Test with a confidence interval of 95\% in GraphPad Prism 5 (GraphPad Software, Inc.) and using ANOVA in STATISTICA 7.0 (Statsoft, Tulsa, OK, USA).

\section{Non-linear Regression Analysis}

Equations 6 and 8 were fitted to data by non-linear regression, using STATISTICAT 7.0 (Statsoft. Inc, USA). The LevenbergMarquardt algorithm for the least squares function minimization was applied. The quality of the regressions was evaluated on the basis of the determination coefficient, $R^{2}$, the squared root mean square error, RMSE (i.e., the square root of the sum of the squared residues (SSE) divided by the regression degrees of freedom) and residuals visual inspection for randomness and normality. $R^{2}$ and SSE were obtained directly from the software. The precision of the estimated parameters was evaluated by the standardized halved width (SHW \%), which was defined as the ratio between the $95 \%$ standard error (obtained from the software) and the value of the estimate (Pinheiro et al. 2015).

\section{Results and Discussion}

\section{Curcumin Nanosystems}

\section{Characterization of the Curcumin Nanoemulsion}

The use of the high-pressure homogenization method resulted in curcumin-loaded nanoemulsions, with a final mean droplet 
diameter of $80.0 \pm 0.9 \mathrm{~nm}$. Regarding $P d I$ and $Z p$, the obtained values were $0.177 \pm 0.009$ and $-65.8 \pm 5.8 \mathrm{mV}$, respectively. The highly negative $Z p$ can be explained by the fact that SDS, an anionic surfactant, was used to stabilize the nanoemulsions (Silva et al. 2015b).

The theoretical minimum size of the curcumin nanoemulsions was calculated using Eq. 1. For this calculation was assumed a surface load of SDS of $1 \mathrm{mg} / \mathrm{m}^{2}$ (BertonCarabin, Ropers, \& Genot 2014), obtaining a minimum theoretical droplet diameter of $67 \mathrm{~nm}$, while the experimental mean droplet diameter obtained for the curcumin-loaded nanoemulsion was $80 \mathrm{~nm}$. This result suggests not only that the concentration of $1 \%(\mathrm{w} / \mathrm{w})$ of SDS used was sufficient to cover all droplets but also that SDS quickly adsorbed on the droplet interface during the homogenization process, which was capable of generating enough disruptive forces (Qian \& McClements, 2011; Silva et al. 2015b; Troncoso et al. 2012). In a previous work (Silva et al. 2015b), where the authors prepared nanoemulsions using the same equipment and process conditions but a nozzle with an orifice of $200-\mu \mathrm{m}$ diameter instead of $100 \mu \mathrm{m}$, a mean droplet size of $137 \mathrm{~nm}$ was obtained, being $57 \mathrm{~nm}$ larger than the value of $80 \mathrm{~nm}$ obtained in this work. The nozzle geometry of is in fact of major importance when developing nanoemulsions (Donsì, Sessa, \& Ferrari 2011), since the reduction of the nozzle diameter allows the generation of stronger disruptive forces, which lead to a reduction in $H_{d}$.

The encapsulation efficiency (EE) and loading capacity (LC) of curcumin using nanoemulsions were determined using Eqs. 2 and 3. The values of EE and LC obtained were $99.8 \pm 0.8 \%$ and $0.53 \pm 0.03 \%$, respectively. The value obtained for the EE is quite good and in line with the results presented by other authors. Sari et al. (2015) prepared curcumin-loaded nanoemulsions using whey protein isolate and Tween 80, achieving an EE of $90.65 \%$ and droplet sizes of $142 \mathrm{~nm}$ (Sari et al. 2015). Friedrich et al. (2015) reported an EE of $100 \%$ for curcumin nanocapsules developed with sorbitan monostearate and poly ( $\varepsilon$-caprolactone) (Friedrich et al. 2015). The low LC may be related with the fact that for the same total volume, small droplet sizes will correspond to a higher number of droplets, with a higher surface area, being the curcumin distributed by this higher number of droplets, thus reducing the content of curcumin within the droplets so that $1 \mathrm{mg}$ of nanoemulsion corresponds to $5.3 \mu \mathrm{g}$ of curcumin.

\section{Polyelectrolytes Adsorption onto Nanoemulsions}

The influence of chitosan concentration was initially evaluated on the properties of the nanoemulsion with the first layer, i.e., $H_{d}, P d I$, and $Z p$. The values of $H_{d}$ and $Z p$ of the multilayer nanoemulsion (first layer) were measured immediately after production (Fig. 1). For the concentrations ranging between
0.02 and $0.05 \%(\mathrm{w} / \mathrm{w})$, these properties were also evaluated after $24 \mathrm{~h}$ of storage. Figure 1al shows that the addition of chitosan leads to a change of $Z p$ values, where the increase of chitosan concentrations (from 0.0005 to $0.08 \%$ ) leads to higher $Z p$ values (from negative to positive). This change reached a positive constant value around $+16 \mathrm{mV}$, when the concentration achieves values ranged between 0.04 and $0.08 \%(\mathrm{w} / \mathrm{w})$. This change in the $Z p$ values suggests that the polyelectrolytes effectively adsorbed on the surface of the nanoemulsion, depositing the first layer of the multilayer emulsion (Szczepanowicz et al. 2015). At chitosan concentrations ranging from 0.0025 to $0.015 \%(\mathrm{w} / \mathrm{w})$, a few "clumps" and oily droplets were observed at the upper surface of the samples. This can be explained by the insufficient mass of chitosan present to coat all the nanoemulsions, which is reflected in the $Z p$ with a decrease from -68.5 to $39.9 \mathrm{mV}$ and consequent aggregation. Charged polyelectrolytes are known to promote bridging flocculation, if there is insufficient chitosan to completely coat the nanoemulsion droplets (Cui et al. 2014; Guzey \& McClements 2006; Mora-Huertas, Fessi, \& Elaissari 2010).

Despite the shift of the electrical charge from negative to positive values when the chitosan concentration changes from 0.05 to $0.08 \%(\mathrm{w} / \mathrm{w})$, some clumps were observed. The increase of chitosan concentrations (from 0.09 to $0.2 \%, \mathrm{w} / \mathrm{w}$ ) promoted the presence of large clumps, making impossible to measure $H_{d}, P d I$, and $Z p$ for nanoemulsions coated with this range of chitosan concentrations. From 0.05 to $0.2 \%$ (w/w), chitosan induced a depletion attraction between the nanoemulsion droplets that lead to the flocculation of particles (Cui et al. 2014; Guzey \& McClements 2006; Szczepanowicz et al. 2015). For the concentration of $0.04 \%$ (w/w), despite the low electrical charge observed in this study, the samples were stable during $24 \mathrm{~h}$ (data not shown), explained by electrostatic and steric repulsions. Therefore, the concentration of $0.04 \%$ $(\mathrm{w} / \mathrm{w})$ of chitosan was selected to carry on the development of the 1st layer.

Sodium alginate, an anionic polyelectrolyte, was used for the development of the 2nd layer, which was deposited on the chitosan layer (1st layer). Increasing the concentrations of alginate (from 0.01 to $0.06 \%, \mathrm{w} / \mathrm{w}$ ) happens a switch in the electrical charge, from positive to negative, reaching a constant negative value around $-50 \mathrm{mV}$ (Fig. 1b1), values that are maintained for alginate concentrations $\geq 0.04 \%(\mathrm{w} / \mathrm{w})$. This suggests that the anionic alginate molecules adsorbed to the surfaces of the 1st layer (chitosan) until they became saturated with alginate (Fig. 1b1).

From Fig. 1b2, it is possible to see that the increase of alginate concentrations leads to an increase of the mean droplet diameter, from $110 \mathrm{~nm}$ (1st layer) to $128 \mathrm{~nm}(0.1 \% \mathrm{w} / \mathrm{w}$ of alginate). The increase of alginate concentration (from 0 to $0.1 \%, \mathrm{w} / \mathrm{w}$ ) also leads to higher values of $P d I$, from 0.177 to 0.231 without and with $0.1 \%(\mathrm{w} / \mathrm{w})$ of alginate, respectively, 
a1)
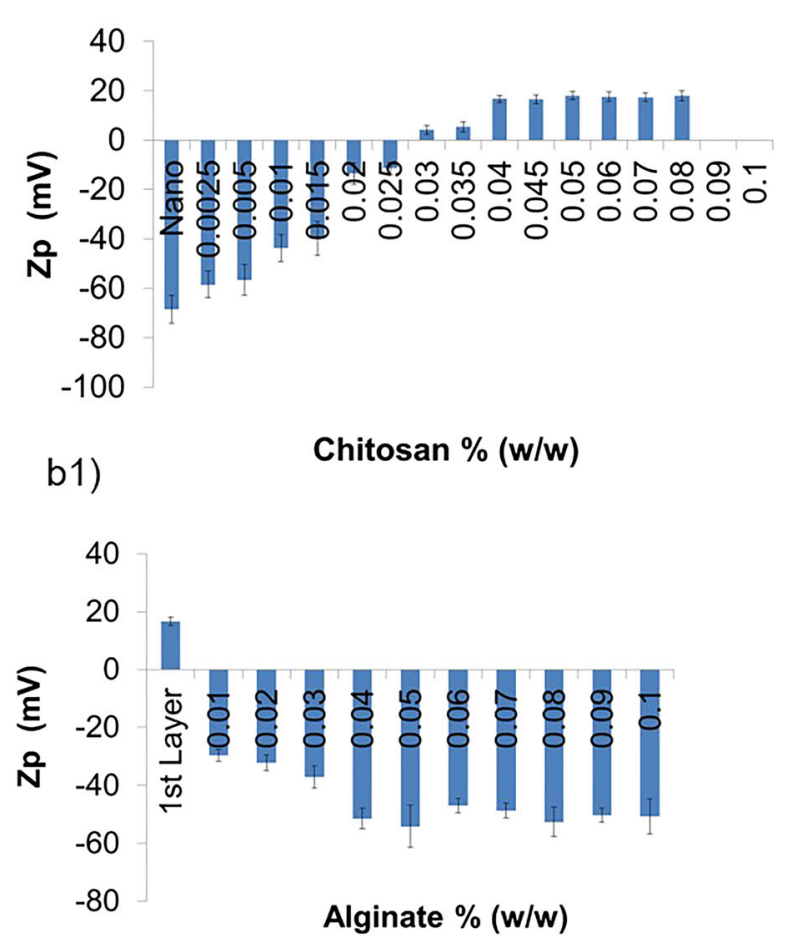

c1)

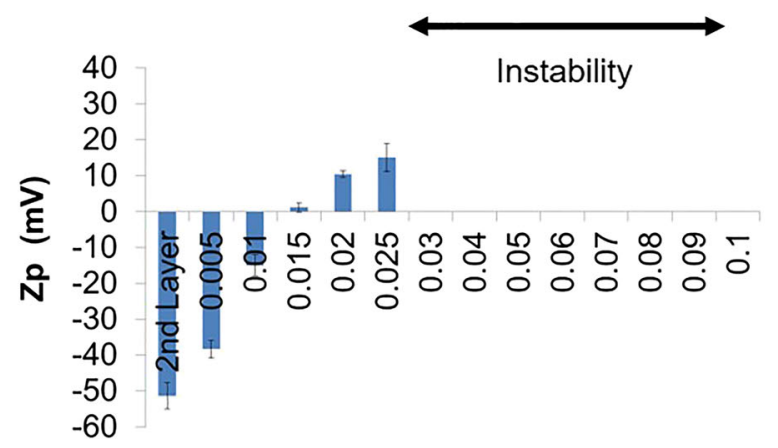

Chitosan \% (w/w)

Fig. 1 Values of a1, b1, and c1 zeta potential $(Z p)$, and a2, b2, and c2 hydrodynamic diameter $\left(H_{d}\right)$, and polydispersity index $(P d I)$ (a) as function of chitosan concentrations for nanoemulsions coated with the 1 st

suggesting that the nanoemulsions were saturated with sodium alginate, due to strong electrostatic repulsions between the multilayer nanoemulsions ( $\mathrm{Li}$ et al. 2010; Pinheiro et al. 2016). Hence, $0.04 \%(\mathrm{w} / \mathrm{w})$ of alginate was selected for the preparation of the 2 nd layer, since the chitosan-SDS-stabilized multilayer nanoemulsions were completely surrounded by alginate molecules, without significant excess of polyelectrolytes in solution.

The results of adding chitosan to build the $3 \mathrm{rd}$ and last layer are shown in Fig. 1c. Chitosan concentrations ranging from 0.01 to $0.1 \%(\mathrm{w} / \mathrm{w})$ were tested. However, for chitosan's a2)

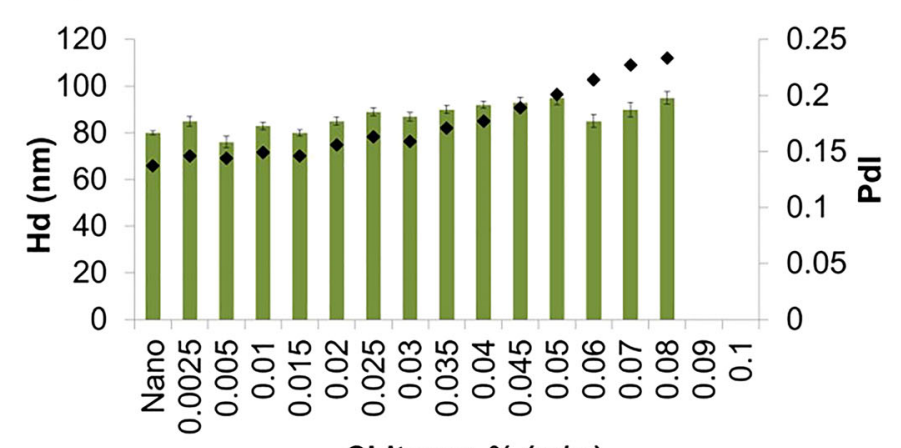

b2)

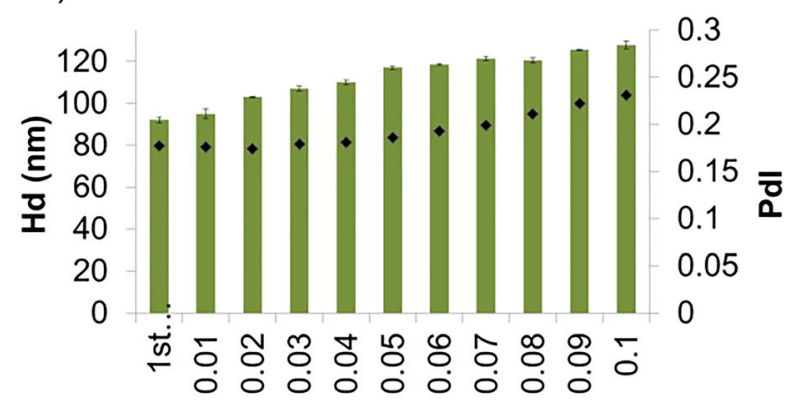

Alginate $\%(w / w)$

c2)

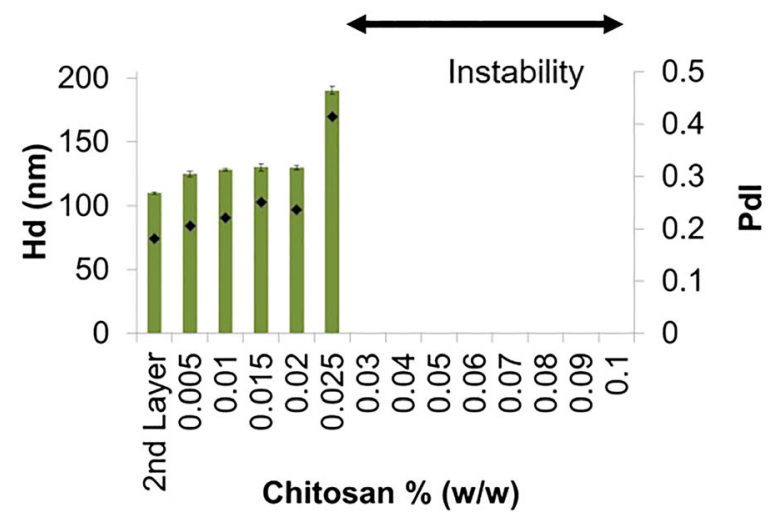

layer; (b) as function of alginate concentrations for nanoemulsions coated with the 2nd layer, and (c) as function of chitosan concentrations for nanoemulsions coated with the 3 rd layer

concentrations $\geq 0.03 \%(\mathrm{w} / \mathrm{w})$, large yellowish clumps were formed, surrounded by a transparent aqueous phase, making it impossible to evaluate $H_{d}, P d I$, and $Z p$ of the developed systems. Due to the presence of a high concentration of free chitosan in the continuous phase, chitosan induced depletion flocculation, aggregating the particles (Cui et al. 2014; Guzey $\&$ McClements 2006; Szczepanowicz et al. 2015). Figure 1c1 shows that at a concentration of $0.015 \%(\mathrm{w} / \mathrm{w})$ of chitosan, neutralization of the electrical charge occurred. At this concentration, there was enough chitosan adsorbed on the anionic groups of alginate capable of neutralizing the anionic groups 
on the adsorbed alginate layer ( $\mathrm{Li}$ et al. 2010). The positively charged molecules of chitosan continued to adsorb to the surface of the SDS-Chitosan-Alginate multilayer nanoemulsions until the charges became positive, up to a certain level of saturation at $0.025 \%(\mathrm{w} / \mathrm{w})$, despite attaining higher values of $H_{d}$ and $P d I$ when compared to the ones obtained at $0.02 \%(\mathrm{w} / \mathrm{w})$ (see Fig. 1c2). At this concentration, a critical electrical charge was reached $(+11 \mathrm{mV})$, opposing to further adsorption due to electrostatic repulsion between the template and the non-adsorbed polyelectrolyte. Therefore, a chitosan concentration of $0.02 \%(\mathrm{w} / \mathrm{w})$ was selected to build the $3 \mathrm{rd}$ layer.

Transmission electron microscopy (TEM) confirmed the values of the mean droplet diameters measured by DLS, supporting the hypothesis of successful multilayer deposition. Figure $2 \mathrm{a}$ and $\mathrm{b}$ show the TEM microphotographs of the nanoemulsion and multilayer nanoemulsion, respectively. Figure $2 \mathrm{~b}$ shows the affinity that uranyl ions have towards chitosan, evidencing the successful development of the multilayer nanoemulsion (Tegge, 1989).

\section{Evaluation of the Interactions Between Polyelectrolytes and Nanoemulsion}

Figure 3 shows the consecutive decrease of frequency after the alternate immersion of the QCM crystal in nanoemulsion, chitosan, alginate, and chitosan solutions, which means that the mass of polyelectrolytes is being successfully deposited. In addition, Fig. 3 shows that the adsorption of alginate results in higher changes of frequency leading to a higher mass deposition, when compared to chitosan. This can be related with the more hydrophilic character of alginate: its deposition can be accompanied by solvent entrapment, resulting in a more viscoelastic system. On the other hand, a more hydrophobic surface, such as the chitosan layer, often does not wet and air can be entrapped, resulting in a smaller measured mass. Similar behavior was observed for alginate/chitosan multilayers (Martins, Mano, \& Alves 2010) for chitosan/fucoidan multilayer nanocapsules (Pinheiro et al. 2015) and lactoferrinGMP nanohydrogels (Bourbon et al. 2015).
When mixing oils, surfactants, bioactive compounds, and polyelectrolytes, physical bonds and chemical interactions are reflected by changes in characteristic bonds, which can be evaluated by FTIR. The characteristic bands for curcumin, SDS, MCTs, chitosan, and sodium alginate are presented in Table 1. Figure 4 shows that the FTIR spectrum of the nanoemulsions majorly presents the characteristic spectra of the MCTs. Nevertheless, from comparing the FTIR profile for the curcumin nanoemulsion with that of pure curcumin and of curcumin in MCTs (data not shown), it is possible to observe that a shift in the out-ofplane bending $\mathrm{CH}$ of aromatic and skeletal $\mathrm{CCH}$ occurred, from $855 \mathrm{~cm}^{-1}$ in the pure curcumin, to $872 \mathrm{~cm}^{-1}$ in the curcumin + MCTs and to $836 \mathrm{~cm}^{-1}$ when encapsulated into the nanoemulsion (Kim et al. 2013). In addition, a shift also occurred in the bending vibrations of $\mathrm{CH}$ bond of alkene group $\left(\mathrm{RCH}=\mathrm{CH}_{2}\right)$ from $962 \mathrm{~cm}^{-1}$ in the pure curcumin to $969 \mathrm{~cm}^{-1}$ in the nanoemulsion. The nanoemulsion presents a peak at $1017 \mathrm{~cm}^{-1}$ when compared to the curcumin in MCTs profile. The in-plane bending of the $\mathrm{CH}_{2}$ group at $1465 \mathrm{~cm}^{-1}$ indicates a reduction of side-by-side chain interactions and an increase in chain motion, which is normally associated with the SDS in the liquid state (Viana, da Silva, \& Pimentel 2012). This indicates that the SDS membrane is surrounding the droplets of oil with encapsulated curcumin. The stretching vibration of the $\mathrm{CO}$ ester groups present in the MCTs also exhibited a slight shift from 1153 to 1154 $\mathrm{cm}^{-1}$ and from 1222 to $1224 \mathrm{~cm}^{-1}$.

With the adsorption of chitosan for the construction of the 1st layer, the FTIR spectrum shows three new peaks at 1045,1649 , and a broad band at $3408 \mathrm{~cm}^{-1}$, that correspond to characteristic peaks of chitosan, skeletal vibration of the $\mathrm{C}-\mathrm{O}$ stretching, carbonyl $(\mathrm{C}=\mathrm{O})$ stretching of the secondary amide (amide I band), and to the amine and hydroxyl groups, respectively (Lawrie et al. 2007; Li, Dai, Zhang, Wang, \& Wei 2008; Pawlak \& Mucha, 2003). Also, the shift of some characteristic peaks of the oil phase, such as the stretching vibration of the $\mathrm{C}-\mathrm{O}$ ester groups from 1154 and 1222 to 1159 and $1218 \mathrm{~cm}^{-1}$ and the SDS in-plane bending of the $\mathrm{CH}_{2}$ group shifted from 1465
Fig. 2 TEM microphotograph of negatively stained nanosystems with uracyl $1 \% \mathrm{w} / \mathrm{w}$. (a) nanoemulsion at $50000 \times$ and (b) multilayer nanoemulsion (three layers) at $100000 \times$

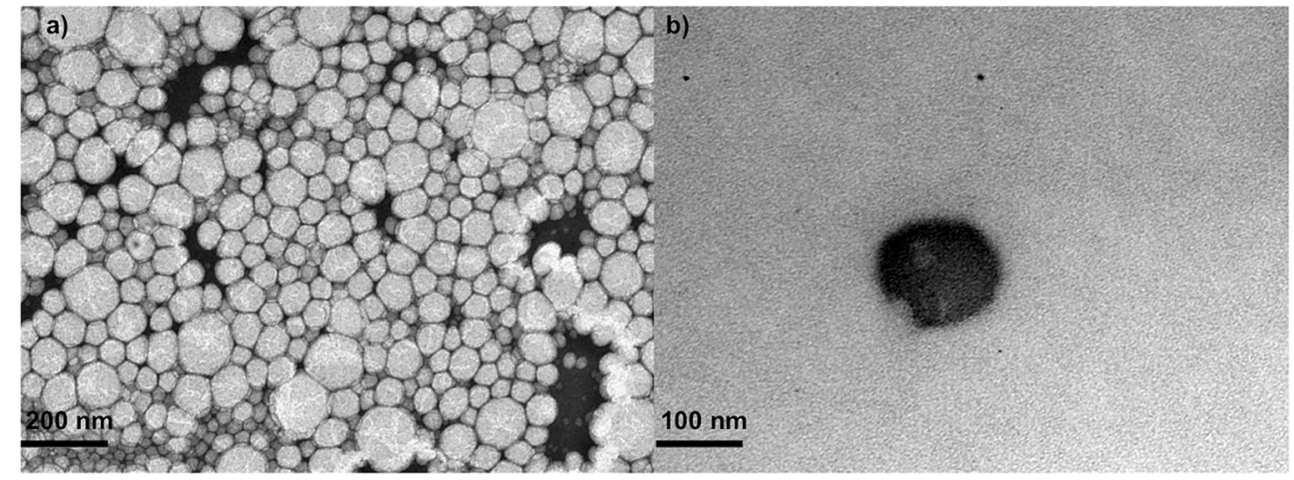


Fig. 3 Real-time monitoring of polyelectrolytes onto nanoemulsion, QCM response signal (resonant frequency, $\Delta F$ ) for the sequential adsorption of nanoemulsion, chitosan, alginate, and chitosan onto a gold electrode surface

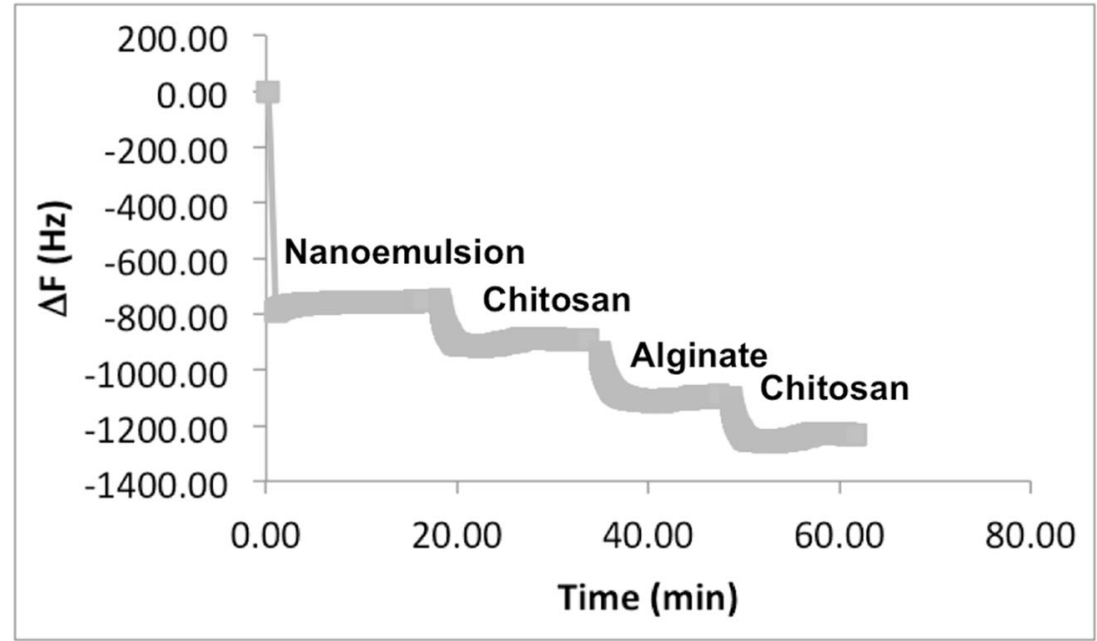

to $1460 \mathrm{~cm}^{-1}$ reveals that chitosan is now the outer layer (Lawrie et al. 2007; Viana et al. 2012; Vlachos et al. 2006; Yang, Irudayaraj, \& Paradkar 2005).

The deposition of alginate as the 2 nd layer was characterized by two new peaks at 1080 and $1631 \mathrm{~cm}^{-1}$, responsible for the asymmetrical stretch of the $\mathrm{C}-\mathrm{O}-\mathrm{C}$ and an asymmetrical stretch vibration of the carboxyl groups (COO), respectively (Lawrie et al. 2007; Shi, Du, Sun, Zhang, \& Dou 2006). The deposition of alginate also leads to a shift in the skeletal vibration of the $\mathrm{C}-\mathrm{O}$ stretching vibration from 1045 to 1055 $\mathrm{cm}^{-1}$ and to a small shoulder at $3455 \mathrm{~cm}^{-1}$ due $\mathrm{OH}$ stretching vibration (Lawrie et al. 2007).

After the development of the 3rd layer, it is possible to observe that residual lactic acid is evident at $1710 \mathrm{~cm}^{-1}$, which corresponds to carbonyl vibration of the carboxylic acid (Bourbon et al. 2011; Lawrie et al. 2007). There is also a shift in the in the skeletal vibration of the $\mathrm{C}-\mathrm{O}$ stretching from 1055 to $1043 \mathrm{~cm}^{-1}$ and a shift in the carbonyl $(\mathrm{C}=\mathrm{O})$ stretching of the secondary amide (amide I band) from 1649 to $1643 \mathrm{~cm}^{-1}$. Additionally, the $\mathrm{OH}$ stretching vibration band of the 3 rd layer was broader and shifted to a lower wave number, $3386 \mathrm{~cm}^{-1}$, suggesting that intermolecular hydrogen bonds also existed in the multilayer nanoemulsions (Shi et al. 2006). It is then plausible to assume that hydrogen bonding can also play an important role in the LbL assembly (Martins et al. 2010).

\section{Evaluation of Temperature and pH Responsiveness}

The thermal stress tests performed on the nanosystems (see "Evaluation of temperature and $\mathrm{pH}$ responses" in Materials and Methods section) after heating the samples from 10 to $80{ }^{\circ} \mathrm{C}$ do not showed any macroscopic sign of instability (i.e., creaming or phase separation). The same behavior was observed by the $H_{d}$ values, that were determined immediately after nanoemulsion preparation and following the $30 \mathrm{~min}$ of heating at each temperature $\left(10\right.$ to $80{ }^{\circ} \mathrm{C}$, please refer to
Table 2). Also in this case, the temperature increase did not have an immediate effect in the characteristics of the nanosystems. Table 2 shows that thermal stress did not influence significantly $(p>0.05)$ the $H_{d}$. This can be explained by the fact that nanoemulsions and multilayer nanoemulsions do not change their morphology, size, and shape as a function of temperature, having low sensibility to the increase of the temperature. Temperature changes did not promote changes in the droplet size, being the droplet size stable to temperature changes during the time of the experiment (Anton \& Vandamme 2011; Gordon, Marom, \& Magdassi 2014; Silva et al. 2015b).

From Fig. 5, it is possible to note that changes in $\mathrm{pH}$ values did not affect the nanoemulsion $H_{d}$. A similar behavior was observed by Tang, Manickam, Wei, and Nashiru (2012), which developed a nanoemulsion loaded with aspirin using Cremophore EL through ultrasound cavitation, obtaining droplet sizes between 200 and $300 \mathrm{~nm}$ and a $P d I$ of about 0.30 . These nanoemulsions were subjected to changes in the $\mathrm{pH}$, revealing to be stable under the range of $\mathrm{pH}$ tested (2-9), even at $\mathrm{pH}$ values close to their isoelectric point, 2.54 (Tang et al. 2012).

The $H_{d}$ of the nanoemulsions with the 2 nd layer and $3 \mathrm{rd}$ layer increased when the $\mathrm{pH}$ was below 3 and above 7 (Fig. $5)$, with a larger increase in $H_{d}$ for the multilayer nanoemulsion with 3 rd layers, from 150 to $200 \mathrm{~nm}$. The increase of $H_{d}$ at acidic $\mathrm{pH}$ (below 3 ) can be attributed to the anionic groups of alginate, which are carboxylic acids with $\mathrm{pK}_{\mathrm{a}}$ values around 3.5 (Harnsilawat, Pongsawatmanit, \& McClements 2006; Li et al. 2010). Nevertheless, alginate layer desorbs from the droplet surfaces in the multilayer system at acidic conditions, even though the electrical charges were supposed to be nearly $0 \mathrm{mV}$. This effect might be due the fact that alginate layer retained some negative charges at $\mathrm{pH}$ below the $\mathrm{pK}_{\mathrm{a}}$. Interestingly, the same behavior was found for $\mathrm{pH}$ above 7 , i.e., above the $\mathrm{pK}_{\mathrm{a}}$ of chitosan, reported to be 


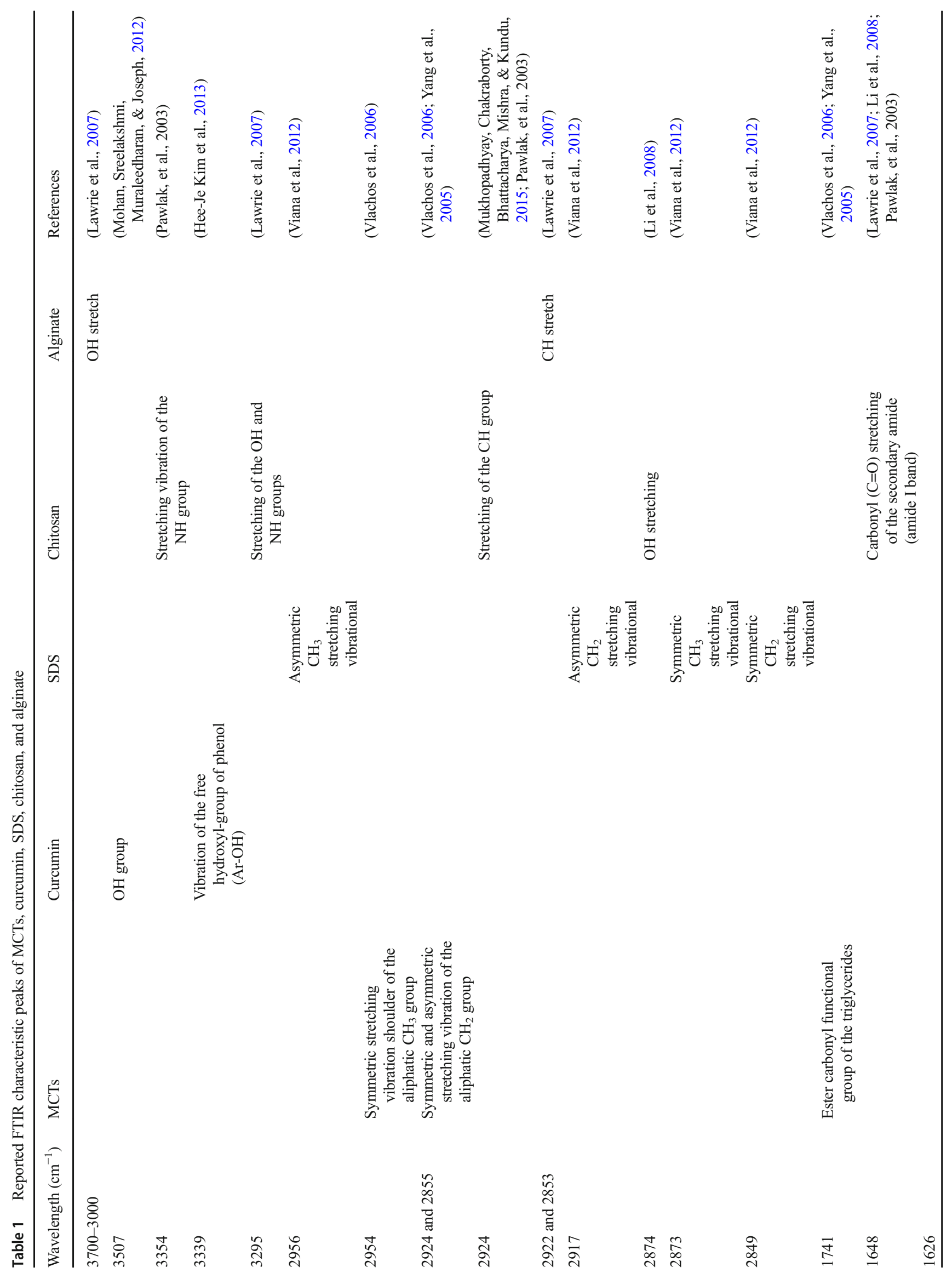



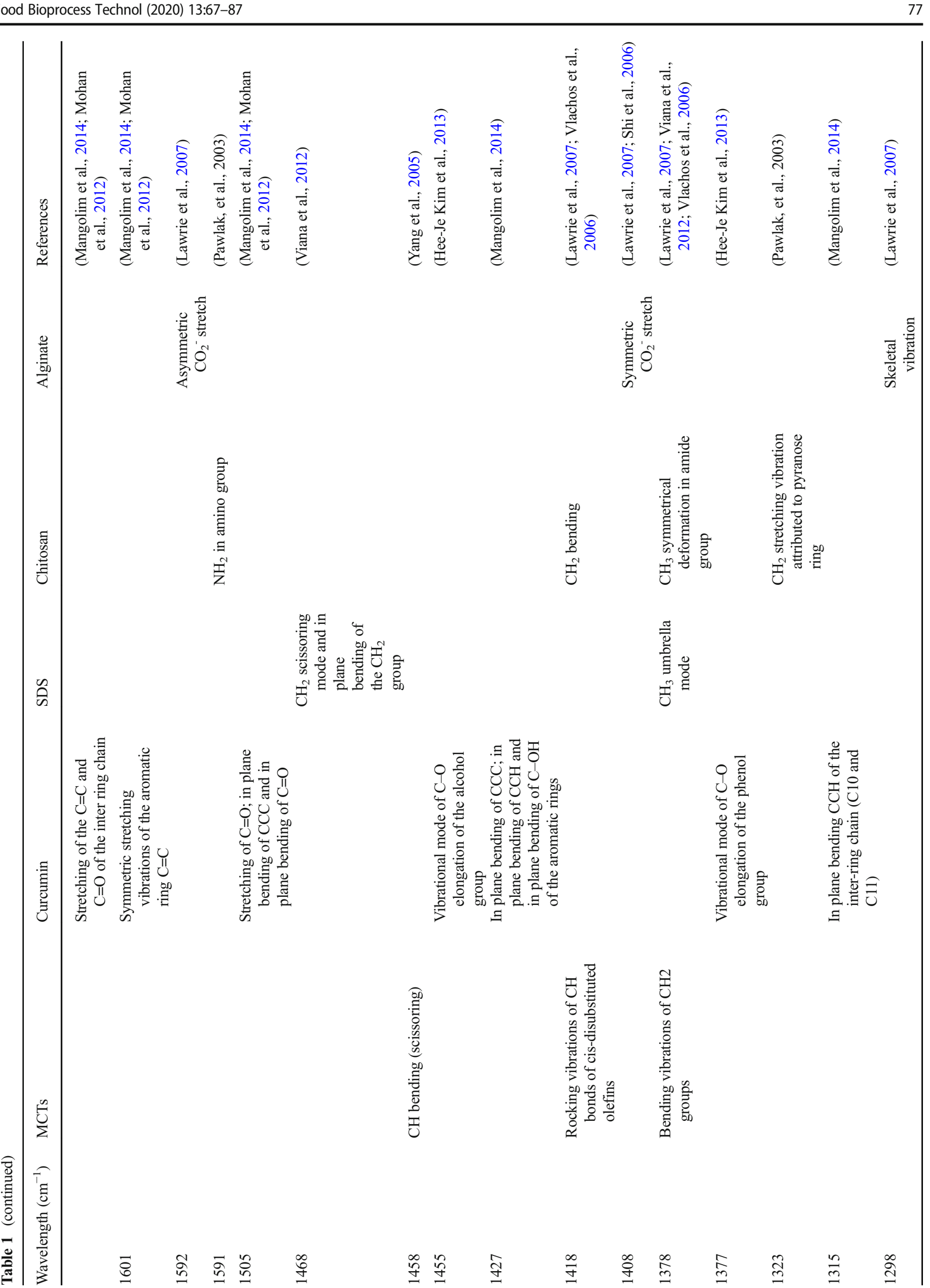


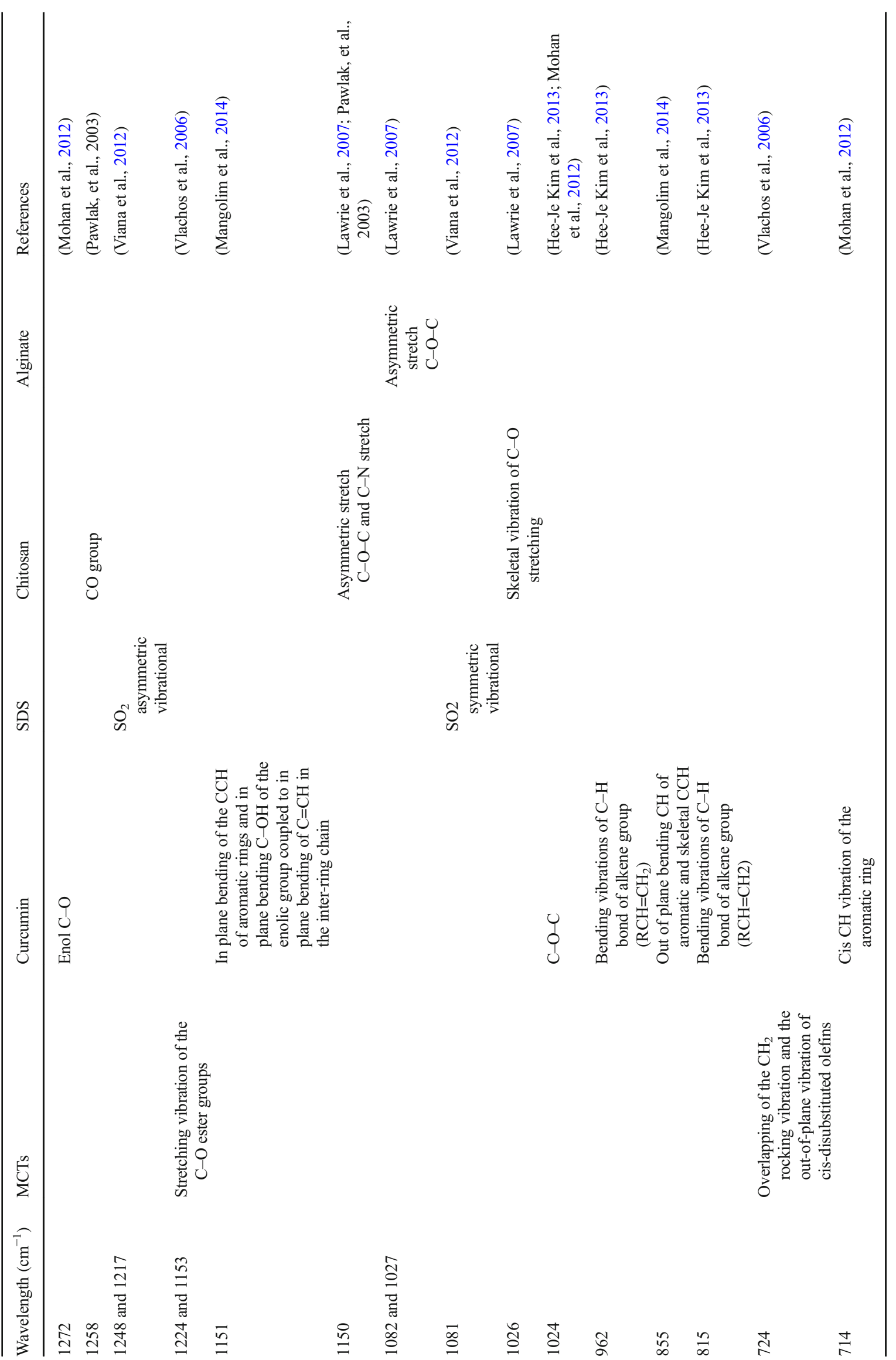




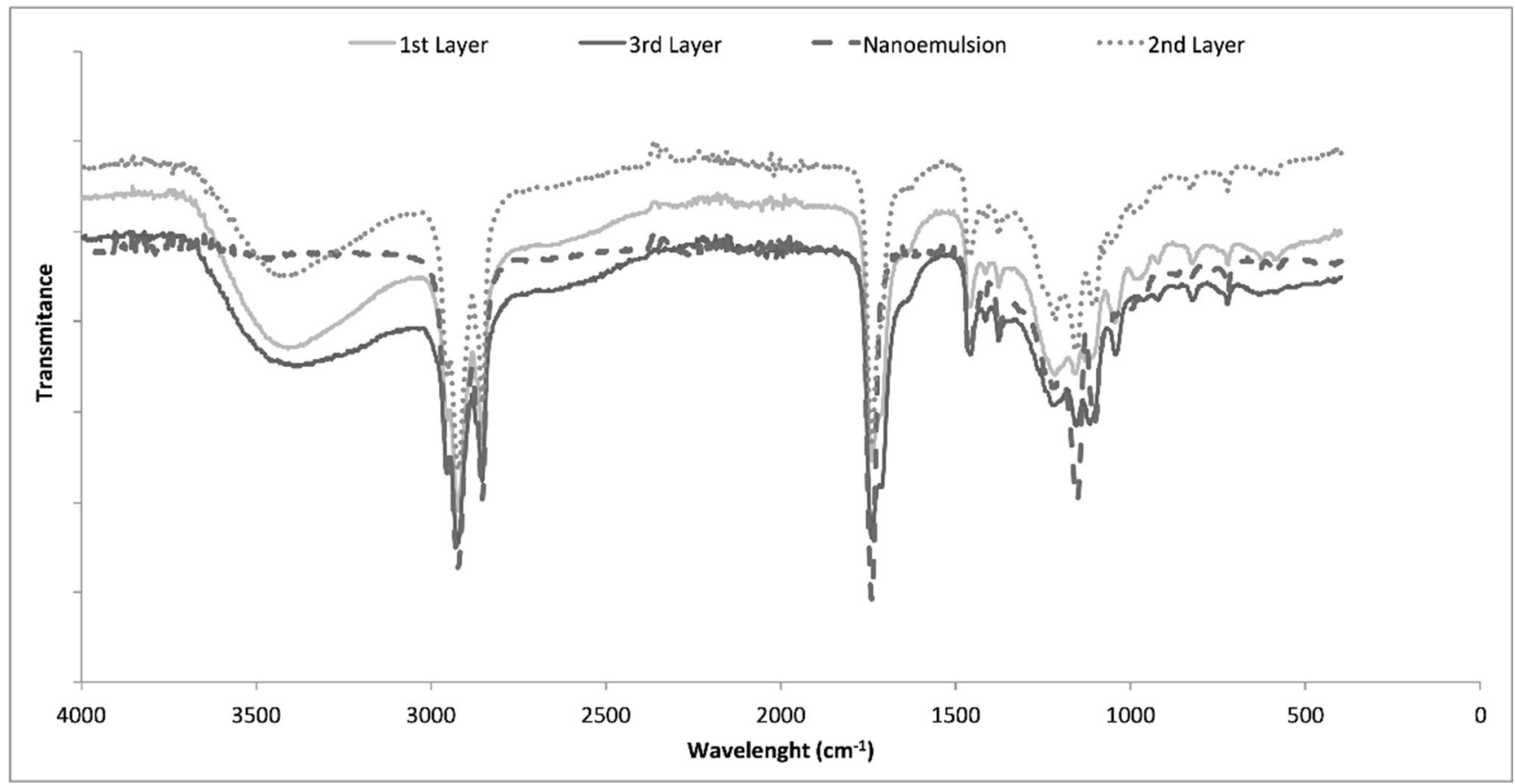

Fig. 4 FTIR spectra of nanoemulsions, 1st layer nanoemulsion; 2nd layer nanoemulsion and 3rd layer nanoemulsion (multilayer nanoemulsion).

around 6.5. At basic $\mathrm{pH}$, the amino groups are completely deprotonated and then contribute to the loss of solubility of the chain segments (Vachoud, Zydowicz, \& Domard 2000). Nevertheless, it can be presumed that the chitosan layers retained some positive charge at neutral and basic $\mathrm{pH}$ values. This is corroborated by previous research that on polyelectrolytes, the $\mathrm{pK}_{\mathrm{a}}$ values of charged groups can change when they are entangled between two oppositely charged polyelectrolytes. This behavior leads to an increasing stability of the multilayer system to variation of $\mathrm{pH}$ (Burke \& Barrett 2003a, b; Li et al. 2010). Despite the increase in the $H_{d}$ for the multilayer nanoemulsion when varying $\mathrm{pH}$, no sign of instability phenomena (i.e., creaming or phase separation) was observed.

\section{Antioxidant Activity}

The antioxidant activity of curcumin encapsulated in the nanosystems was not significantly different from that of free curcumin in MCT, when determined by DPPH tests. The radical scavenging activity for the un-encapsulated curcumin was $9.4 \pm 0.5 \mu \mathrm{mol} \mathrm{L}{ }^{-1}$ Trolox $/ \mathrm{mg}$ curcumin, while for the nanoemulsion, it was $9.4 \pm 0.3 \mu \mathrm{mol} \mathrm{L}{ }^{-1}$ Trolox $/ \mathrm{mg}$ curcumin and for the multilayer nanoemulsion, it was $9.3 \pm 0.6 \mu \mathrm{mol}$ $\mathrm{L}^{-1}$ Trolox/mg curcumin. Sari et al. (2015) produced curcumin nanoemulsions using whey protein isolate, where 10 to $40 \mathrm{mg}$ of curcumin was added to $100 \mathrm{~mL}$ of total emulsion and achieved a lower radical scavenging activity in the order of $3.33 \pm 0.02 \mu \mathrm{mol} \mathrm{L}{ }^{-1}$ Trolox $/ \mathrm{mg}$ of curcumin for the
Table 2 Experimental $H_{d}$ and $P d I$ values obtained for the nanosystems after thermal stress tests

\begin{tabular}{|c|c|c|c|c|}
\hline \multirow[b]{2}{*}{ Treatment } & \multicolumn{2}{|c|}{ Nanoemulsion } & \multicolumn{2}{|c|}{ Multilayer nanoemulsion } \\
\hline & $H_{d}(\mathrm{~nm})$ & $P d I$ & $H_{d}(\mathrm{~nm})$ & $P d I$ \\
\hline After production & $80.0 \pm 1.6^{\mathrm{a}}$ & $0.137 \pm 0.04^{\mathrm{a}}$ & $129.8 \pm 3.2^{\mathrm{a}}$ & $0.237 \pm 0.03^{\mathrm{a}}$ \\
\hline $10^{\circ} \mathrm{C}$ & $80.8 \pm 1.7^{\mathrm{a}}$ & $0.134 \pm 0.02^{\mathrm{a}}$ & $125.2 \pm 2.1^{\mathrm{a}}$ & $0.235 \pm 0.02^{\mathrm{a}}$ \\
\hline $20{ }^{\circ} \mathrm{C}$ & $81.2 \pm 1.6^{\mathrm{a}}$ & $0.131 \pm 0.03^{\mathrm{a}}$ & $125.1 \pm 2.8^{\mathrm{a}}$ & $0.241 \pm 0.02^{\mathrm{a}}$ \\
\hline $30{ }^{\circ} \mathrm{C}$ & $82.8 \pm 2.1^{\mathrm{a}}$ & $0.133 \pm 0.03^{\mathrm{a}}$ & $125.8 \pm 2.7^{\mathrm{a}}$ & $0.236 \pm 0.01^{\mathrm{a}}$ \\
\hline $40{ }^{\circ} \mathrm{C}$ & $83.3 \pm 1.5^{\mathrm{a}}$ & $0.137 \pm 0.03^{\mathrm{a}}$ & $124.7 \pm 2.9^{\mathrm{a}}$ & $0.242 \pm 0.02^{\mathrm{a}}$ \\
\hline $50{ }^{\circ} \mathrm{C}$ & $82.7 \pm 1.7^{\mathrm{a}}$ & $0.133 \pm 0.02^{\mathrm{a}}$ & $125.7 \pm 3.2^{\mathrm{a}}$ & $0.239 \pm 0.02^{\mathrm{a}}$ \\
\hline $60{ }^{\circ} \mathrm{C}$ & $82.6 \pm 1.9^{\mathrm{a}}$ & $0.134 \pm 0.02^{\mathrm{a}}$ & $124.3 \pm 3.3^{\mathrm{a}}$ & $0.238 \pm 0.01^{\mathrm{a}}$ \\
\hline $70{ }^{\circ} \mathrm{C}$ & $82.9 \pm 1.9^{\mathrm{a}}$ & $0.129 \pm 0.04^{\mathrm{a}}$ & $125.8 \pm 2.4^{\mathrm{a}}$ & $0.237 \pm 0.03^{\mathrm{a}}$ \\
\hline $80{ }^{\circ} \mathrm{C}$ & $84.7 \pm 3.1^{\mathrm{a}}$ & $0.134 \pm 0.02^{\mathrm{a}}$ & $128.5 \pm 2.9^{\mathrm{a}}$ & $0.241 \pm 0.03^{\mathrm{a}}$ \\
\hline
\end{tabular}

Each value represents mean $\pm \mathrm{SD}(n=3)$. Different letters between rows mean statistically different results $(p<$ $0.05)$ 
Fig. 5 pH responsiveness of the nanosystems in terms of hydrodynamic diameter $\left(H_{d}\right)$

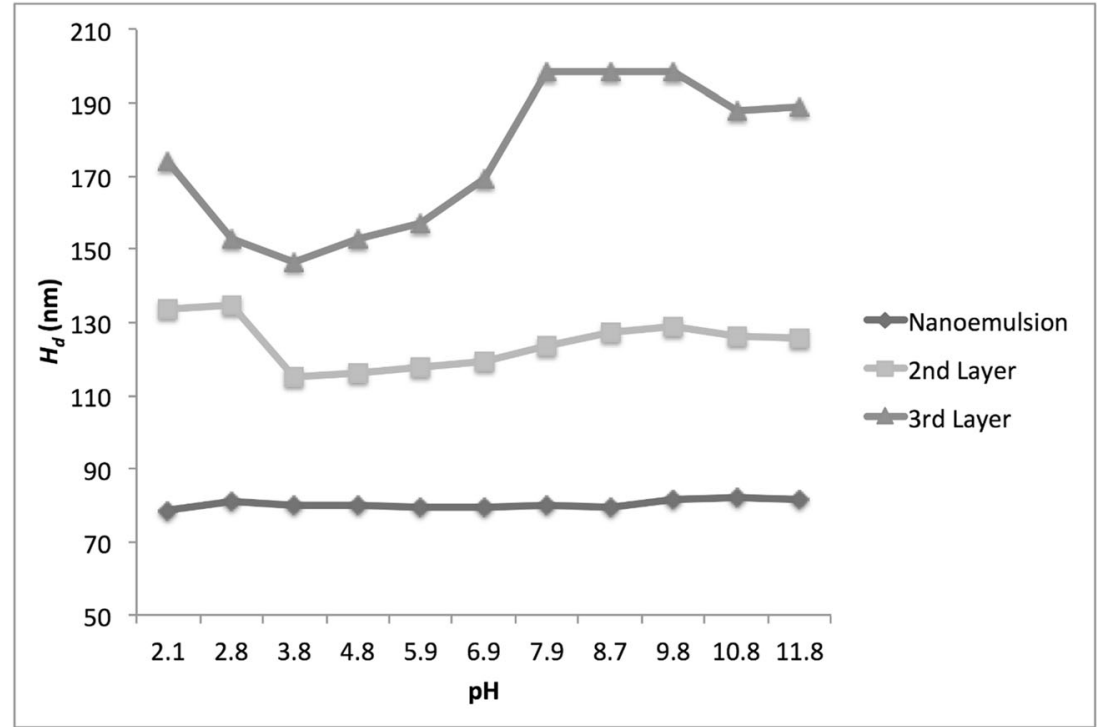

nanoemulsion system, whereas for native curcumin, the value was $3.53 \pm 0.11 \mu \mathrm{mol} \mathrm{L}{ }^{-1}$ Trolox/mg of curcumin. In the present study, the radical scavenging activity for the nanosystems was measured during 35 days of storage, at $4{ }^{\circ} \mathrm{C}$ and 25 ${ }^{\circ} \mathrm{C}$ and in the absence of light (A.L) and in the presence of light (P.L). Table 3 shows that curcumin is degraded during storage, since the radical scavenging activity (RSA) decreases during storage. From results present in Table 3, it is possible to see that samples that were kept in the dark had a significantly lower $(p<0.05)$ decrease in the RSA when compared to the ones that were kept under light after 28 days and 14 days when stored at $4{ }^{\circ} \mathrm{C}$ and $25^{\circ} \mathrm{C}$, respectively. This was expected due the fast degradation of curcumin when exposed to light (Khurana \& Ho, 1988). For the nanoemulsions, losses between $18.7 \pm 1.2$ and $25.5 \pm 1.5 \%$ were observed when samples were kept in the dark at $4{ }^{\circ} \mathrm{C}$ and exposed to light at 25 ${ }^{\circ} \mathrm{C}$. For pure curcumin solubilized in MCTs in the same conditions, a decrease in the RSA of $21.9 \pm 1.4 \%$ and $26.6 \pm 1.1 \%$ was observed. Despite lipids and surfactants are known for significantly decreasing curcumin degradation, it was expected that nanoencapsulation decreased the degradation of curcumin (Priyadarsini 2009). Contrary to what was expected, the development of layers around the nanoemulsion did not decrease the RSA during storage, on the contrary, a significantly decrease in RSA during storage was observed after 7 days of storage (Table 3 ). The addition of consecutive layers led to a decrease in the antioxidant activity of the curcumin, especially in the presence of light at $25^{\circ} \mathrm{C}$. It is a known fact that curcumin is very sensitive to light, $\mathrm{pH}$, solvent system, and oxygen (Siviero et al. 2015). For the 1st layer, the decrease of RSA when exposed to light and at $25{ }^{\circ} \mathrm{C}$ was of $43.7 \pm 1.7 \%$ against the decrease of $26.9 \pm 1.3 \%$ when stored in the dark at the same temperature. For the 3rd layer, the same behavior was observed, achieving a loss of $73.8 \pm 1.2 \%$ at 25
${ }^{\circ} \mathrm{C}$ in the presence of light against $66.0 \pm 1.8 \%$ when stored in the dark. It was possible to follow this degradation visually, since the solution turned colorless for the $3 \mathrm{rd}$ layer at $25^{\circ} \mathrm{C}$ in the presence of light. The processes behind curcumin degradation are very complex, because polarity, $\pi$-bonding nature, hydrogen bond donating, and accepting properties of the solvent influence the excited state photophysics of curcumin (Priyadarsini, 2009). Tomren et al. (2007) showed that curcumin in cyclodextrin solutions was more resistant to hydrolysis than free curcumin; nevertheless, curcumin in cyclodextrin showed to be equally or more susceptible to photochemical degradation, depending on the medium. They observed that stability depends either on the presence of organic solvents or the absence of water (Tomren et al. 2007). Nevertheless, the difference between the RSA decrease for the nanoemulsion and the multilayer nanoemulsion (3rd layer) was around 50\% higher for the multilayer nanoemulsion. This could be explained by the difference in the aqueous medium. The multilayer nanoemulsion was developed using the saturation method that generates massive dilutions; each layer deposition increases the dilution, performing a 8-fold dilution, achieving a final $\mathrm{pH}$ of 5 , which excludes degradation of curcumin to neutral $\mathrm{pH}$. This difference in the aqueous phase volume could lead to higher hydrogen binding ability, promoting the degradation of curcumin (Priyadarsini, 2009). The hydrogen donation reaction from curcumin leads to the oxidation of curcumin (Priyadarsini, 2014).

\section{Curcumin Release from the Nanosystems}

The release behavior of the curcumin from the developed nanosystems was evaluated at $4{ }^{\circ} \mathrm{C}, 25{ }^{\circ} \mathrm{C}$, and 37 ${ }^{\circ} \mathrm{C}$ in four different acceptor media, i.e., ethanol at 10 , 
20 , and $50 \%(\mathrm{v} / \mathrm{v})$ and SDS at $5 \%(\mathrm{v} / \mathrm{v})$, always maintaining the sink conditions for curcumin.

Ethanol at 10 and $20 \%(\mathrm{v} / \mathrm{v})$ solutions are food simulants (as defined by the European Commission on the Official Journal of the European Union) assigned for foods that have a hydrophilic character and are able to extract hydrophilic substances. Ethanol at 20\% (v/v) shall be used for alcoholic foods with an alcohol content of up to $20 \%$ and for those foods that contain a relevant amount of organic ingredients that render the food more lipophilic. In contrast, food simulants like ethanol at $50 \%(\mathrm{v} / \mathrm{v})$ are assigned for foods that have a lipophilic character and are able to extract lipophilic substances. Ethanol at $50 \%(\mathrm{v} / \mathrm{v})$ shall be used for alcoholic foods with an alcohol content above $20 \%$ and for oil-in-water emulsions (EC, 2011).

Concerning the release tests using 10 and $20 \%(\mathrm{v} / \mathrm{v})$ of ethanol as the acceptor medium at $4{ }^{\circ} \mathrm{C}$ and $25^{\circ} \mathrm{C}$, it was observed that both nanosystems were unable to release curcumin (data not shown), maintaining the curcumin entrapped within the nanosystems. These nanosystems also showed $H_{d}$ stability during the period of the release assays (5 days), whereas the mean droplet diameter was $80.0 \pm$ $0.9 \mathrm{~nm}$ and $130.1 \pm 1.5 \mathrm{~nm}$ for the nanoemulsion and multilayer nanoemulsion, respectively, before the assay, being after the release assay $79.2 \pm 1.5$ and $127.3 \pm 2.8 \mathrm{~nm}$, respectively, when using $10 \%(\mathrm{v} / \mathrm{v})$ of ethanol. For $20 \%(\mathrm{v} / \mathrm{v})$ of ethanol, the $H_{d}$ after the assays was $77.2 \pm 2.4$ and $125.4 \pm 3.9 \mathrm{~nm}$, respectively, for the nanoemulsion and multilayer nanoemulsion. The stability of the nanosystems in ethanol $20 \%(\mathrm{v} / \mathrm{v})$ during 5 days of storage provides an indication of which food products are best suited for the incorporation of the developed nanosystems (e.g., mayonnaise, mustard, salad creams, ice creams, non-alcoholic beverages and alcoholic beverages up to $20 \%, \mathrm{v} / \mathrm{v}$ ), nevertheless further studies should be performed to confirm the trend of this indication.

For the conditions of $50 \%(\mathrm{v} / \mathrm{v})$ ethanol at $25{ }^{\circ} \mathrm{C}$ and $5 \%$ (v/v) SDS at $25^{\circ} \mathrm{C}$ and $37^{\circ} \mathrm{C}$, the LSM fitting curves adequately describe the experimental data (Fig. 6) achieving a good regression quality $\left(R^{2}>0.87\right)$, being most parameters estimated with good precision. This suggests that this model can be used to describe the transport mechanisms involved in curcumin release for both nanosystems. From the parameters presented in Table 4, it can be seen that Brownian motion of curcumin in the nanosystems cannot describe the transport mechanism for itself, i.e., it does not strictly follow Fick's behavior, being governed by both Fickian and Case II transport, with only one main relaxation of the nanosystem (i.e., surfactant and polyelectrolytes relaxation). Figure 6 a shows that the release of curcumin from nanoemulsions, when using SDS as the acceptor medium at $37^{\circ} \mathrm{C}$, happens during the first $10 \mathrm{~h}$, releasing a total mass of curcumin of $\approx 100 \mu \mathrm{g}$, which was 2.5 times higher when compared to the release of curcumin in $5 \% \mathrm{v} / \mathrm{v}$ SDS at $25{ }^{\circ} \mathrm{C}$ (maximum curcumin 
a)

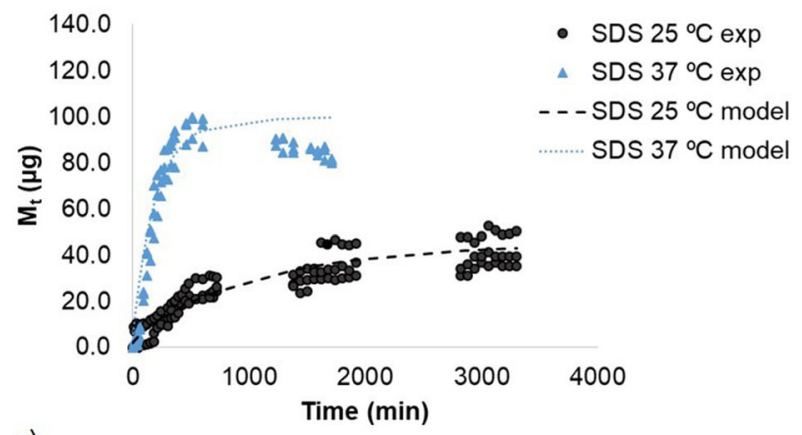

c)

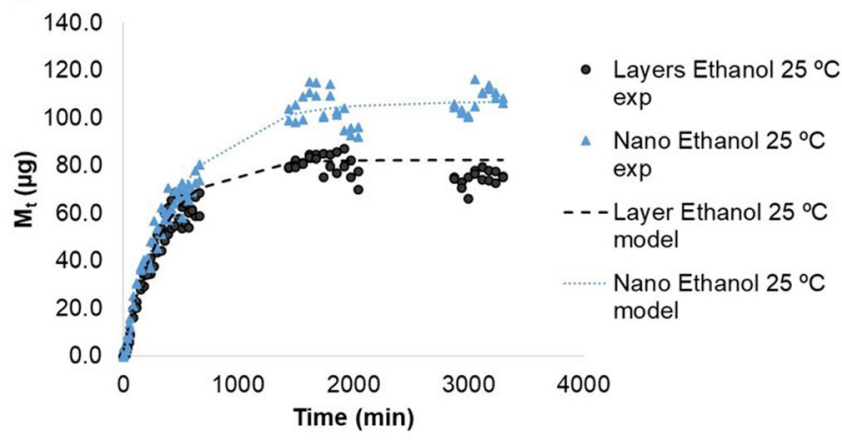

Fig. 6 Profile of curcumin release from nanosystems: (a) effect of temperature, using 5\% (w/w) SDS as acceptor medium; (b) effect of polyelectrolytes at $25{ }^{\circ} \mathrm{C}$ and $37{ }^{\circ} \mathrm{C}$, using $5 \%(w / w)$ SDS as acceptor

release of $\approx 40 \mu \mathrm{g}$ ). Also the release of curcumin from the nanoemulsion in $5 \% \mathrm{v} / \mathrm{v}$ SDS at $25{ }^{\circ} \mathrm{C}$ is much slower, happening during $48 \mathrm{~h}$ before achieving a stagnant phase. Data in Table 4 show that relaxation is the main mechanism of transport, since it is responsible for $90 \%$ of the curcumin released, $M_{R}$, while Brownian motion is responsible for only $10 \%, M_{F}$.

The relaxation effect is understood as a time-dependent phenomenon with a characteristic time constant that reflects the reformation process of the surface layer (Atsumi, Akiko, Keiji, \& Kenshiro 1994). Briefly, there are two relaxation processes involved in surfactant micellar solutions. The first is a fast relaxation time, associated with the collision of surfactant monomers with micelles, generally in the order of the microseconds. The second relaxation time is related to the micelle formation and dissolution process, being an indicator of the micelle stability (Atsumi et al. 1994). b)

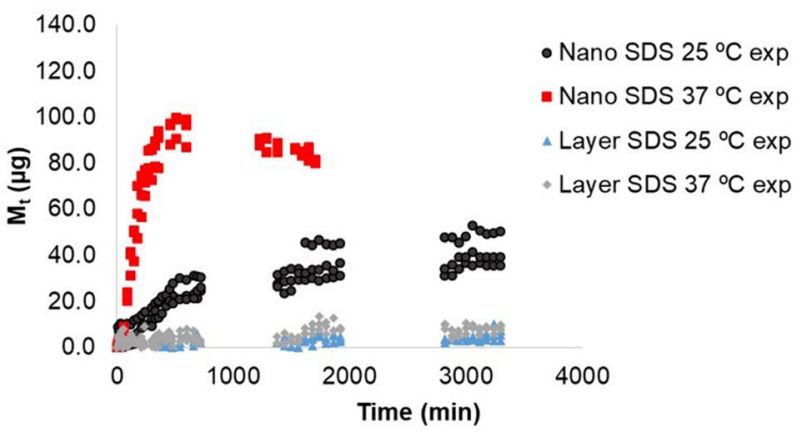

d)

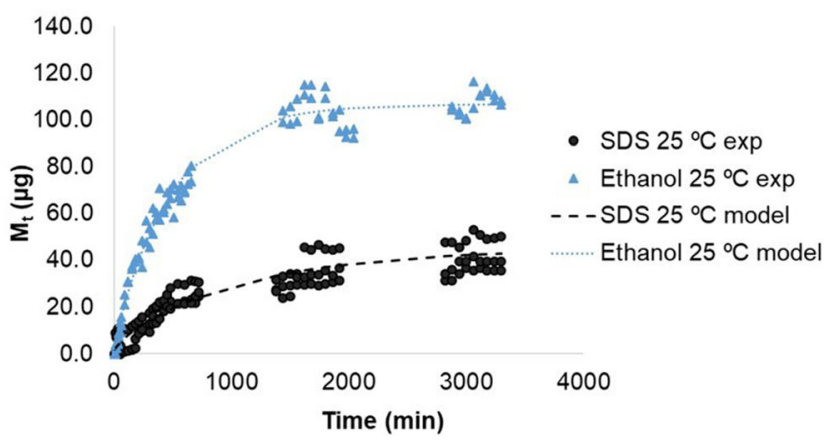

medium; (c) effect of polyelectrolytes at $25{ }^{\circ} \mathrm{C}$, using $50 \%(\mathrm{v} / \mathrm{v})$ ethanol as acceptor medium; (d) effect of acceptor medium (ethanol vs SDS) at 25 ${ }^{\circ} \mathrm{C}$

Surfactant molecules maintain an equilibrium surface concentration (nanoemulsion stability) through a continuous dynamic exchange with surfactant monomers (and possibly larger assemblies such as surfactant micelles) in the continuous phase (Mason, Wilking, Meleson, Chang, \& Graves 2006). Due to this, in the presence of high concentrations of SDS, nanoemulsion droplets can quickly exchange monomers with the surfactant monomers or even micelles, promoting the release of curcumin (Atsumi et al. 1994; Mason et al. 2006; Paruchuri, Nalaskowski, Shah, \& Miller 2006).

The effect of temperature on the mass released by relaxation can be explained by the fact that a higher temperature leads to a decrease in the second relaxation rate that may promote instability, while leading to a decrease in the first relaxation time (Atsumi et al. 1994; Milcovich \& Asaro 2012; Paruchuri et al. 2006). In addition, it is known that for

Table 4 Results of fitting the LSM to experimental data of the curcumin release profile. Evaluation of the quality of the regression on the basis of $R^{2}$

\begin{tabular}{|c|c|c|c|c|c|c|c|c|}
\hline Nanosystem & Acceptor medium & $\mathrm{T}\left({ }^{\circ} \mathrm{C}\right)$ & $R^{2}$ & $M_{F}(\mu \mathrm{g})$ & $K_{F}\left(\mathrm{~cm}^{-1}\right)$ & $M_{R}(\mu \mathrm{g})$ & $K_{R}\left(\mathrm{~cm}^{-1}\right)$ & $M_{T}(\mu \mathrm{g})$ \\
\hline \multirow[t]{2}{*}{ Nanoemulsion } & \multirow[t]{2}{*}{ SDS } & 25 & 0.8758 & 4.44 & 0.0047 & 36.69 & 0.0012 & 41.13 \\
\hline & & 37 & 0.9280 & 10.00 & 0.0010 & 90.97 & 0.0054 & 100.97 \\
\hline Nanoemulsion & $50 \%$ vol. ethanol & 25 & 0.9889 & 5.78 & 0.014 & 100.98 & 0.0020 & 106.76 \\
\hline Multilayer nanoemulsion & $50 \%$ vol. ethanol & 25 & 0.9845 & 5.00 & 0.0029 & 77.37 & 0.0029 & 82.37 \\
\hline
\end{tabular}

$T$, temperature; $M_{F}$, equilibrium amount of sorption in unrelaxed polymer; $M_{R}$, equilibrium sorption of the relation process; $K_{F}$, fick rate constant; $K_{R}$, relaxation rate constant 
higher temperatures, the solubility of bioactive compounds could be increased, which can be associated with the decrease in the first relaxation time and trigger a higher amount of $M_{R}$, contributing to a slower rate of release. The multilayer nanoemulsion system was unable to release curcumin maintaining it entrapped (Fig. 6b). Here, the addition of polyelectrolyte layers reinforced the stability of these structures, avoiding curcumin release, probably by inhibiting the exchange of monomers with the surfactant monomers or micelles from the acceptor medium. For assays at $37^{\circ} \mathrm{C}$, a possible explanation for the release behavior is the fact that alginate and chitosan exhibit a gel form, which can further prevent the release of curcumin (Azevedo et al. 2014; Carreira, Gonçalves, Mendonça, Gil, \& Coelho 2010). The effect of polyelectrolyte layers in the release of curcumin was also evaluated, at $50 \%(\mathrm{v} / \mathrm{v})$ of ethanol at $25^{\circ} \mathrm{C}$. From the parameters presented in Table 4, it can be observed that relaxation was the main phenomenon responsible for curcumin release $\left(M_{R}>M_{F}\right)$. Ethanol is a water-soluble solvent that has the ability to modify the bulk physicochemical properties of aqueous solutions, such as density, refractive index, and interfacial tension. At the same time, ethanol is able to alter the aqueous solutions structural properties, such as the optimum curvature, solubility, and phase behavior (Saberi, Fang, \& McClements 2013). Due to this, ethanol may induce surfactant and polymer relaxation. Ethanol is also known for its ability to create pores; although not fully understood, it may be related to a partial removal of the hydration water between surfactants and polyelectrolytes, resulting in segregation of the surfactant or polyelectrolyte network in the presence of ethanol (Delcea, Möhwald, \& Skirtach 2011). From Fig. 6c, it is possible to observe that coating the nanoemulsion with polyelectrolytes did not slow down the initial burst phase; nevertheless, the final amount of released curcumin was decreases from $M_{T}=$ $82.37 \mu \mathrm{g}$ to $M_{T}=106.76 \mu \mathrm{g}$, for multilayer nanoemulsion and nanoemulsion, respectively. A similar behavior was described by Sharipova et al. (2016), which observed that the building of polymer-surfactant complexes leads to a decrease in the release of $\alpha$-tocopherol, promoting the release of $\alpha$ tocopherol during $80 \mathrm{~h}$ (Sharipova et al. 2016). Table 4 shows that $M_{R}$ of curcumin released from nanoemulsions is higher $(100.98 \mu \mathrm{g})$ than that obtained from multilayer nanoemulsions $(77.37 \mu \mathrm{g})$. The addition of the polyelectrolyte layers may have slowed down the partial removal of the hydration water between surfactants and polyelectrolytes, which resulted in the segregation of the polyelectrolyte network, in the presence of ethanol. Moreover, a solvent like ethanol plays an important role in the establishment of hydrogen bonds (Delcea et al. 2011). As shown in the FTIR section, the structure of multilayer nanoemulsions showed hydrogen bonds between alginate and chitosan that in the presence of ethanol might be disrupted, explaining the lower amount of curcumin released into the acceptor medium.

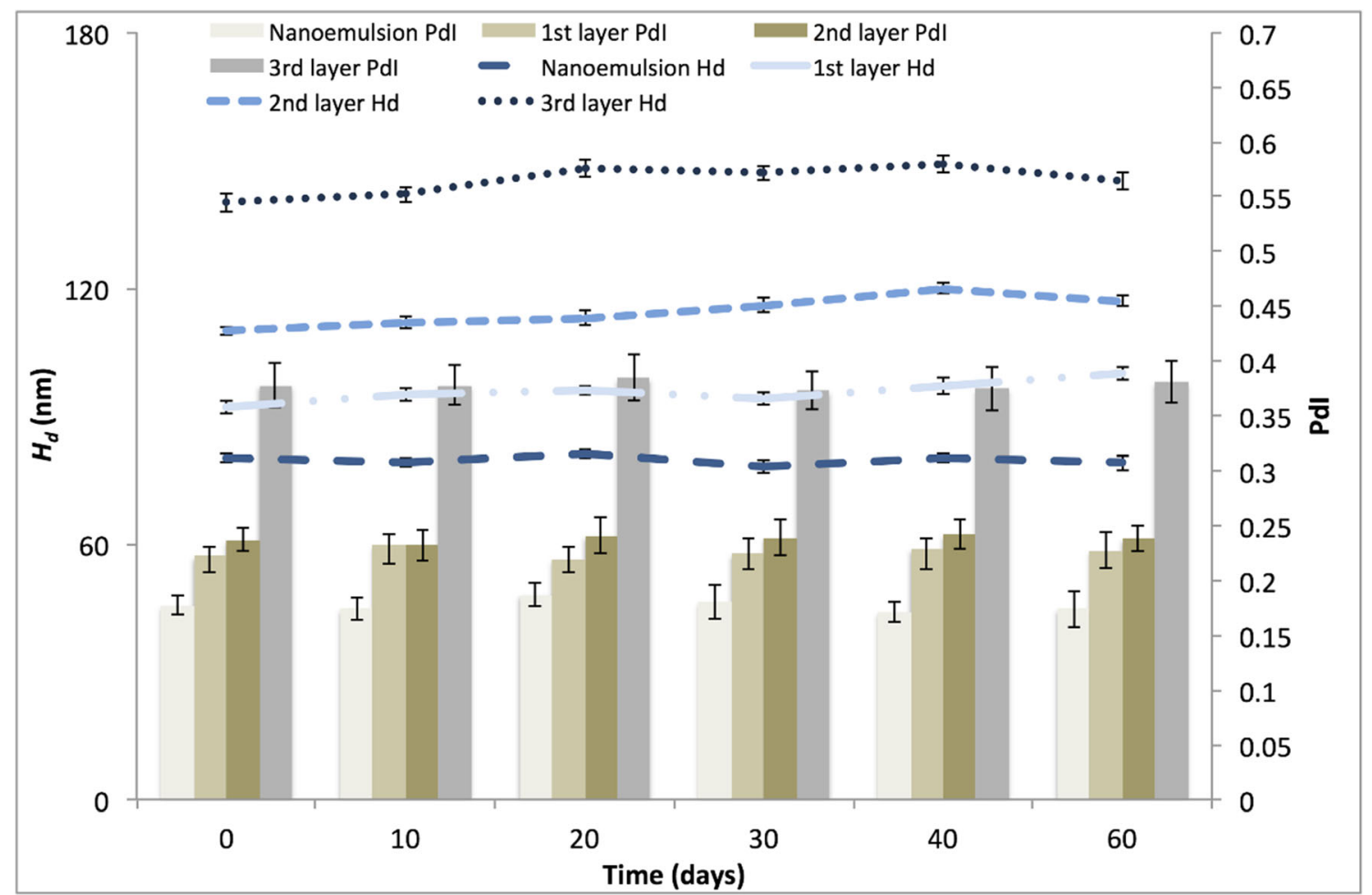

Fig. 7 Hydrodynamic diameter $\left(H_{d}\right)$ and polydispersity index $(P d I)$ during 60 days of storage. Bars indicate standard deviation $(n=3)$. Lines are for readers' guidance and do not represent a model prediction 
The influence of the acceptor medium was also evaluated. The experimental data and parameters present in Fig. 6d and Table 4 show that considerably higher amounts of curcumin were released from the nanoemulsions, when $50 \%(\mathrm{v} / \mathrm{v})$ of ethanol was used as the acceptor medium, achieving a $M_{T}$ of $106.76 \mu \mathrm{g}$, while for the $5 \%(\mathrm{v} / \mathrm{v}) \mathrm{SDS}$, the $M_{T}$ was $41.13 \mu \mathrm{g}$. These differences may be explained by the ability of ethanol to create pores in the nanosystems, increasing the amount of released curcumin, due to the partial removal of hydration water between the surfactants (Delcea et al. 2011).

\section{Nanosystems Stability Under Storage Conditions}

Curcumin nanoemulsions and multilayer nanoemulsions showed no macroscopic sign of instability phenomena (i.e., creaming or phase separation) after 3 months of storage. The results showed that curcumin nanoemulsions and multilayer nanoemulsions maintained their values of $H_{d}$ and $P d I$ (Fig. 7). Results showed that after storage, $H_{d}$ values did not present statistically significant differences $(p>0.05)$ (data not shown) when compared to the values obtained immediately after production (Fig. 7).

\section{Conclusions}

This work shows that layer-by-layer assembly could successfully change the surface characteristics of nanoemulsions. These nanosystems showed stability under storage conditions in terms of $H_{d}$ and $P d I$, being stable to changes in temperature and $\mathrm{pH}$-responsiveness. From quartz crystal microbalance measurements, it is possible to conclude that deposition of chitosan and alginate results in the formation of a stable multilayer structure, mainly due electrostatic interactions between the polyelectrolytes, while FTIR showed that interactions such as hydrogen bonds might also be involved. Fitting the linear superimposition model to experimental data of curcumin release suggested an anomalous behavior, being relaxation of the polymers/surfactant the main transport phenomenon observed. This work clarifies the release mechanism involved when two different acceptor media are used, enlightens the impact of temperature, solvent and surface properties in the controlled release. This work also suggests that these nanosystems may be used in different food and/or beverage products with great potential to act as controlled delivery systems for lipophilic bioactive compounds.

Acknowledgments The authors Hélder D. Silva and Ana C. Pinheiro (SFRH/BD/81288/2011, SFRH/BPD/101181/2014, respectively) are the recipients of a fellowship from the Fundação para a Ciência e Tecnologia (FCT, Portugal). The authors would like to acknowledge Rui Fernandes from IBMC, University of Porto, for assistance in taking the TEM pictures and Estefanía López Silva, from CACTI, University of Vigo for assistance in the FTIR analysis. The authors thank the FCT Strategic
Project PEst-OE/EQB/LA0023/2013 and the project "BioIndBiotechnology and Bioengineering for improved Industrial and AgroFood processes," REF.NORTE-07-0124- FEDER-000028, co-funded by the Programa Operacional Regional do Norte (ON.2 - O Novo Norte), QREN, FEDER. We also thank the European Commission: BIOCAPS (316265, FP7/REGPOT-2012-2013.1). This work was supported by the "CARINA" project for the safeness, sustainability, and competitiveness of agro-food productions of Campania Region. The support of EU Cost Action FA1001 is gratefully acknowledged. The authors also acknowledge Stepan for providing the Neobee 1053 oil.

\section{References}

Adamczak, M., Kupiec, A., Jarek, E., Szczepanowicz, K., \& Warszyński, P. (2014). Preparation of the squalene-based capsules by membrane emulsification method and polyelectrolyte multilayer adsorption. Colloids and Surfaces A: Physicochemical and Engineering Aspects, 462, 147-152.

Anton, N., \& Vandamme, T. (2011). Nano-emulsions and micro-emulsions: clarifications of the critical differences. Pharmaceutical Research, 28(5), 978-985.

Artiga-Artigas M, Lanjari-Pérez Y, Martín-Belloso O. (2018). Curcuminloaded nanoemulsions stability as affected by the nature and concentration of surfactant. Food Chem. 266:466-474. https://doi.org/ 10.1016/j.foodchem.2018.06.043

Atsumi, O., Akiko, M., Keiji, S., \& Kenshiro, T. (1994). Dynamic properties of soluble monolayer of sodium dodecyl sulfate (SDS) on aqueous solution. Japanese Journal of Applied Physics, 33(10B), L1468.

Azevedo, M. A., Bourbon, A. I., Vicente, A. A., \& Cerqueira, M. A. (2014). Alginate/chitosan nanoparticles for encapsulation and controlled release of vitamin B2. International Journal of Biological Macromolecules, 71, 141-146.

Berens, A. R., \& Hopfenberg, H. B. (1978). Diffusion and relaxation in glassy polymer powders: 2 . Separation of diffusion and relaxation parameters. Polymer, 19(5), 489-496.

Berton-Carabin, C. C., Ropers, M.-H., \& Genot, C. (2014). Lipid oxidation in oil-in-water emulsions: involvement of the interfacial layer. Comprehensive Reviews in Food Science and Food Safety, 13(5), 945-977.

Bourbon, A. I., Pinheiro, A. C., Cerqueira, M. A., Rocha, C. M. R., Avides, M. C., Quintas, M. A. C., \& Vicente, A. A. (2011). Physico-chemical characterization of chitosan-based edible films incorporating bioactive compounds of different molecular weight. Journal of Food Engineering, 106(2), 111-118.

Bourbon, A. I., Pinheiro, A. C., Carneiro-da-Cunha, M. G., Pereira, R. N., Cerqueira, M. A., \& Vicente, A. A. (2015). Development and characterization of lactoferrin-GMP nanohydrogels: evaluation of $\mathrm{pH}$, ionic strength and temperature effect. Food Hydrocolloids, 48, 292-300.

Burke, S. E., \& Barrett, C. J. (2003a). Acid-base equilibria of weak polyelectrolytes in multilayer thin films. Langmuir, 19(8), 3297 3303.

Burke, S. E., \& Barrett, C. J. (2003b). pH-responsive properties of multilayered poly(1-lysine)/hyaluronic acid surfaces. Biomacromolecules, 4(6), 1773-1783.

Carreira, A. S., Gonçalves, F. A. M. M., Mendonça, P. V., Gil, M. H., \& Coelho, J. F. J. (2010). Temperature and $\mathrm{pH}$ responsive polymers based on chitosan: applications and new graft copolymerization strategies based on living radical polymerization. Carbohydrate Polymers, 80(3), 618-630.

Cerqueira, M., Pinheiro, A., Silva, H., Ramos, P., Azevedo, M., FloresLópez, M., Rivera, M., Bourbon, A., Ramos, Ó., \& Vicente, A. 
(2014). Design of bio-nanosystems for oral delivery of functional compounds. Food Engineering Reviews, 6(1-2), 1-19.

Choi, A.-J., Kim, C.-J., Cho, Y.-J., Hwang, J.-K., \& Kim, C.-T. (2011). Characterization of capsaicin-loaded nanoemulsions stabilized with alginate and chitosan by self-assembly. Food and Bioprocess Technology, 4(6), 1119-1126.

Cui, J., van Koeverden, M. P., Müllner, M., Kempe, K., \& Caruso, F. (2014). Emerging methods for the fabrication of polymer capsules. Advances in Colloid and Interface Science, 207, 14-31.

Delcea, M., Möhwald, H., \& Skirtach, A. G. (2011). Stimuli-responsive LbL capsules and nanoshells for drug delivery. Advanced Drug Delivery Reviews, 63(9), 730-747.

Donsì, F., Sessa, M., \& Ferrari, G. (2011). Effect of emulsifier type and disruption chamber geometry on the fabrication of food nanoemulsions by high pressure homogenization. Industrial \& Engineering Chemistry Research, 51(22), 7606-7618.

EC. (2011). Plastic materials and articles intended to come into contact with food. In E. Commission (Ed.), Commission Regulation (EU) No 10/2011. Official Journal of the European Union.

EFSA. (2010). Call for scientific data on food additives permitted in the $\mathrm{EU}$ and belonging to the functional classes of emulsifiers, stabilisers and gelling agents.

Ezhilarasi, P. N., Karthik, P., Chhanwal, N., \& Anandharamakrishnan, C. (2013). Nanoencapsulation techniques for food bioactive components: a review. Food and Bioprocess Technology, 6, 628-647.

Food and Drug Administration (2019) 21CFR172.822. Code of federal regulations, title 21 , volume 3 . https://www.accessdata.fda.gov/ scripts/cdrh/cfdocs/cfcfr/CFRSearch.cfm?fr=172.822. Accessed 11 Nov 2019.

Friedrich, R. B., Kann, B., Coradini, K., Offerhaus, H. L., Beck, R. C. R., \& Windbergs, M. (2015). Skin penetration behavior of lipid-core nanocapsules for simultaneous delivery of resveratrol and curcumin. European Journal of Pharmaceutical Sciences, 78, 204-213.

Gordon, V., Marom, G., \& Magdassi, S. (2014). Formation of hydrophilic nanofibers from nanoemulsions through electrospinning. International Journal of Pharmaceutics, 478(1), 172-179.

Guttoff, M., Saberi, A. H., \& McClements, D. J. (2015). Formation of vitamin $\mathrm{D}$ nanoemulsion-based delivery systems by spontaneous emulsification: factors affecting particle size and stability. Food Chemistry, 171(0), 117-122.

Guzey, D., \& McClements, D. J. (2006). Formation, stability and properties of multilayer emulsions for application in the food industry. Advances in Colloid and Interface Science, 128-130, 227-248.

Harnsilawat, T., Pongsawatmanit, R., \& McClements, D. J. (2006). Characterization of $\beta$-lactoglobulin-sodium alginate interactions in aqueous solutions: a calorimetry, light scattering, electrophoretic mobility and solubility study. Food Hydrocolloids, 20(5), 577-585.

Hu, K., Huang, X., Gao, Y., Huang, X., Xiao, H., \& McClements, D. J. (2015). Core-shell biopolymer nanoparticle delivery systems: synthesis and characterization of curcumin fortified zein-pectin nanoparticles. Food Chemistry, 182, 275-281.

Kaur, K., Kumar, R., \& Mehta, S. K. (2015). Nanoemulsion: a new medium to study the interactions and stability of curcumin with bovine serum albumin. Journal of Molecular Liquids, 209, 62-70.

Khurana, A., \& Ho, C.-T. (1988). High performance liquid chromatographic analysis of curcuminoids and their photo-oxidative decomposition compounds in curcuma longa L. Journal of Liquid Chromatography, 11(11), 2295-2304.

Kim, H.-J., Kim, D.-J., Karthick, S. N., Hemalatha, K. V., Raj, C. J., Ok, S., \& Choe, Y. (2013). Curcumin dye extracted from curcuma longa L. Used as sensitizers for efficient dye-sensitized solar cells. International Journal of Electrochemical Science, 8, 8320-8328.

Lawrie, G., Keen, I., Drew, B., Chandler-Temple, A., Rintoul, L., Fredericks, P., \& Grøndahl, L. (2007). Interactions between alginate and chitosan biopolymers characterized using FTIR and XPS. Biomacromolecules, 8(8), 2533-2541.
Lee, S.J., Choi S.J , Li, Y., Decker, E.A., McClements, D.J. (2011) Protein-Stabilized Nanoemulsions and Emulsions: Comparison of Physicochemical Stability, Lipid Oxidation, and Lipase Digestibility. Journal of Agricultural and Food Chemistry, 59, 415-427

Li, P., Dai, Y.-N., Zhang, J.-P., Wang, A.-Q., \& Wei, Q. (2008). Chitosanalginate nanoparticles as a novel drug delivery system for nifedipine. International Journal of Biomedical Science : IJBS, 4(3), 221-228.

Li, Y., Hu, M., Xiao, H., Du, Y., Decker, E. A., \& McClements, D. J. (2010). Controlling the functional performance of emulsion-based delivery systems using multi-component biopolymer coatings. European Journal of Pharmaceutics and Biopharmaceutics, 76(1), $38-47$.

Li, M., Zhang, F., Liu, Z., Guo, X., \& Qiao, L. (2018). Controlled release system by active gelatin film incorporated with $\beta$-cyclodextrinthymol inclusion complexes. Food and Bioprocess Technology, 1-8.

Li, X., Wu, G., Qi, X., Zhang, H., Wang, L., Qian, H. (2019). Physicochemical properties of stable multilayer nanoemulsion prepared via the spontaneously-ordered adsorption of short and long chains. Food Chemistry, 274, 620-628

Liechty, W. B., Scheuerle, R. L., \& Peppas, N. A. (2013). Tunable, responsive nanogels containing t-butyl methacrylate and 2-(tbutylamino)ethyl methacrylate. Polymer, 54(15), 3784-3795.

Liu, Y., Cai, Y., Jiang, X., Wu, J., \& Le, X. (2016). Molecular interactions, characterization and antimicrobial activity of curcumin-chitosan blend films. Food Hydrocolloids, 52, 564-572.

Madrigal-Carballo, S., Lim, S., Rodriguez, G., Vila, A. O., Krueger, C. G., Gunasekaran, S., \& Reed, J. D. (2010). Biopolymer coating of soybean lecithin liposomes via layer-by-layer self-assembly as novel delivery system for ellagic acid. Journal of Functional Foods, 2(2), 99-106.

Malvern, I. (2011). Dynamic light scattering common terms defined. In M. Instruments (Ed.). Worcestershire, UK.

Mangolim, C. S., Moriwaki, C., Nogueira, A. C., Sato, F., Baesso, M. L., Neto, A. M., \& Matioli, G. (2014). Curcumin- $\beta$-cyclodextrin inclusion complex: Stability, solubility, characterisation by FT-IR, FTRaman, X-ray diffraction and photoacoustic spectroscopy, and food application. Food Chemistry, 153, 361-370.

Martins, G. V., Mano, J. F., \& Alves, N. M. (2010). Nanostructured selfassembled films containing chitosan fabricated at neutral $\mathrm{pH}$. Carbohydrate Polymers, 80(2), 570-573.

Mason, T. G., Wilking, J. N., Meleson, K., Chang, C. B., \& Graves, S. M. (2006). Nanoemulsions: formation, structure, and physical properties. Journal of Physics: Condensed Matter, 18(41), R635.

McClements, D. J., \& Xiao, H. (2012). Potential biological fate of ingested nanoemulsions: influence of particle characteristics. Food \& Function, 3(3), 202-220.

Milcovich, G., \& Asaro, F. (2012). Insights into catanionic vesicles thermal transition by NMR spectroscopy. In V. Starov \& P. Griffiths (Eds.), UK Colloids 2011 (Vol. 139, pp. 35-38). Berlin Heidelberg: Springer.

Mohan, P. R. K., Sreelakshmi, G., Muraleedharan, C. V., \& Joseph, R. (2012). Water soluble complexes of curcumin with cyclodextrins: characterization by FT-Raman spectroscopy. Vibrational Spectroscopy, 62, 77-84.

Mora-Huertas, C. E., Fessi, H., \& Elaissari, A. (2010). Polymer-based nanocapsules for drug delivery. International Journal of Pharmaceutics, 385(1-2), 113-142.

Morais Diane, J. M., \& Burgess, J. (2014). Vitamin E nanoemulsions characterization and analysis. International Journal of Pharmaceutics, 465(1-2), 455-463.

Mukhopadhyay, P., Chakraborty, S., Bhattacharya, S., Mishra, R., \& Kundu, P. P. (2015). pH-sensitive chitosan/alginate core-shell nanoparticles for efficient and safe oral insulin delivery. International Journal of Biological Macromolecules, 72, 640-648. 
Ozturk, B., Argin, S., Ozilgen, M., \& McClements, D. J. (2014). Formation and stabilization of nanoemulsion-based vitamin E delivery systems using natural surfactants: Quillaja saponin and lecithin. Journal of Food Engineering, 142(0), 57-63.

Paruchuri, V. K., Nalaskowski, J., Shah, D. O., \& Miller, J. D. (2006). The effect of cosurfactants on sodium dodecyl sulfate micellar structures at a graphite surface. Colloids and Surfaces A: Physicochemical and Engineering Aspects, 272(3), 157-163.

Pawlak, A., \& Mucha, M. (2003). Thermogravimetric and FTIR studies of chitosan blends. Thermochimica Acta, 396(1-2), 153-166.

Pinheiro, A. C., Bourbon, A. I., Quintas, M. A. C., Coimbra, M. A., \& Vicente, A. A. (2012). K-carrageenan/chitosan nanolayered coating for controlled release of a model bioactive compound. Innovative Food Science \& Emerging Technologies, 16(0), 227-232.

Pinheiro, A. C., Bourbon, A. I., Cerqueira, M. A., Maricato, É., Nunes, C., Coimbra, M. A., \& Vicente, A. A. (2015). Chitosan/fucoidan multilayer nanocapsules as a vehicle for controlled release of bioactive compounds. Carbohydrate Polymers, 115, 1-9.

Pinheiro, A. C., Coimbra, M. A., \& Vicente, A. A. (2016). In vitro behaviour of curcumin nanoemulsions stabilized by biopolymer emulsifiers - Effect of interfacial composition. Food Hydrocolloids, 52, 460-467.

Plaza-Oliver, M., Baranda, J. F., Rodríguez Robledo, V., Castro-Vázquez, L., Gonzalez-Fuentes, J., Marcos, P., Lozano, M. V., SantanderOrtega, M. J., \& Arroyo-Jimenez, M. M. (2015). Design of the interface of edible nanoemulsions to modulate the bioaccessibility of neuroprotective antioxidants. International Journal of Pharmaceutics, 490(1-2), 209-218.

Priyadarsini, K. I. (2009). Photophysics, photochemistry and photobiology of curcumin: studies from organic solutions, bio-mimetics and living cells. Journal of Photochemistry and Photobiology C: Photochemistry Reviews, 10(2), 81-95.

Priyadarsini, K. (2014). The chemistry of curcumin: from extraction to therapeutic agent. Molecules, 19(12), 20091-20112.

Qian, C., Decker, E.A., Xiao, H., McClements, D.J.. (2012) Physical and chemical stability of b-carotene-enriched nanoemulsions: Influence of $\mathrm{pH}$, ionic strength, temperature, and emulsifier type. Food Chemistry, 132, 1221-1229

Qian, C., \& McClements, D. J. (2011). Formation of nanoemulsions stabilized by model food-grade emulsifiers using high-pressure homogenization: factors affecting particle size. Food Hydrocolloids, 25(5), 1000-1008.

Rao, J., \& McClements, D. J. (2013). Optimization of lipid nanoparticle formation for beverage applications: influence of oil type, cosolvents, and cosurfactants on nanoemulsion properties. Journal of Food Engineering, 118(2), 198-204.

Rufino, M. d. S. M., Alves, R. E., Brito, E. S. d., Morais, S. M. d., Sampaio, C. d. G., Pérez - Jiménez, J., \& Saura-Calixto, F. D. (2007). Metodologia científica: determinação da atividade antioxidante total em frutas pela captura do radical livre DPPH. In Embrapa Agroindústria Tropical. Comunicado técnico (Ed.), (Vol. 2015). Fortaleza: Embrapa Agroindústria Tropical.

Saberi, A. H., Fang, Y., \& McClements, D. J. (2013). Fabrication of vitamin E-enriched nanoemulsions by spontaneous emulsification: effect of propylene glycol and ethanol on formation, stability, and properties. Food Research International, 54(1), 812-820.

Saberi, A. H., Zeeb, B., Weiss, J., \& McClements, D. J. (2015). Tuneable stability of nanoemulsions fabricated using spontaneous emulsification by biopolymer electrostatic deposition. Journal of Colloid and Interface Science, 455, 172-178.

Sari, T. P., Mann, B., Kumar, R., Singh, R. R. B., Sharma, R., Bhardwaj, M., \& Athira, S. (2015). Preparation and characterization of nanoemulsion encapsulating curcumin. Food Hydrocolloids, 43, 540-546.

Sharipova, A. A., Aidarova, S. B., Grigoriev, D., Mutalieva, B., Madibekova, G., Tleuova, A., \& Miller, R. (2016). Polymer- surfactant complexes for microencapsulation of vitamin $\mathrm{E}$ and its release. Colloids and Surfaces B: Biointerfaces, 137, 152-157.

Shi, X., Du, Y., Sun, L., Zhang, B., \& Dou, A. (2006). Polyelectrolyte complex beads composed of water-soluble chitosan/alginate: characterization and their protein release behavior. Journal of Applied Polymer Science, 100(6), 4614-4622.

Silva, H. D., Cerqueira, M. A., Souza, B. W. S., Ribeiro, C., Avides, M. C., Quintas, M. A. C., Coimbra, J. S. R., Carneiro-da-Cunha, M. G., $\&$ Vicente, A. A. (2011). Nanoemulsions of $\beta$-carotene using a highenergy emulsification-evaporation technique. Journal of Food Engineering, 102(2), 130-135.

Silva, H. D., Cerqueira, M. A., \& Vicente, A. A. (2012). Nanoemulsions for food applications: development and characterization. Food and Bioprocess Technology, 5(3), 854-867.

Silva, H. D., Cerqueira, M. A., \& Vicente, A. A. (2015a). Chapter 56 Nanoemulsion-based systems for food applications. In B. I. Kharisov (Ed.), CRC concise encyclopedia of nanotechnology. Boca Raton: CRC Press by Taylor and Francis Group.

Silva, H. D., Cerqueira, M. A., \& Vicente, A. A. (2015b). Influence of surfactant and processing conditions in the stability of oil-in-water nanoemulsions. Journal of Food Engineering, 167, 89-98.

Siviero, A., Gallo, E., Maggini, V., Gori, L., Mugelli, A., Firenzuoli, F., \& Vannacci, A. (2015). Curcumin, a golden spice with a low bioavailability. Journal of Herbal Medicine, 5(2), 57-70.

Souza, B. W. S., Cerqueira, M. A., Bourbon, A. I., Pinheiro, A. C., Martins, J. T., Teixeira, J. A., Coimbra, M. A., \& Vicente, A. A. (2012). Chemical characterization and antioxidant activity of sulfated polysaccharide from the red seaweed Gracilaria birdiae. Food Hydrocolloids, 27(2), 287-292.

Spigno, G., Donsì, F., Amendola, D., Sessa, M., Ferrari, G., \& De Faveri, D. M. (2013). Nanoencapsulation systems to improve solubility and antioxidant efficiency of a grape marc extract into hazelnut paste. Journal of Food Engineering, 114(2), 207-214.

Szczepanowicz, K., Bazylińska, U., Pietkiewicz, J., Szyk-Warszyńska, L., Wilk, K. A., \& Warszyński, P. (2015). Biocompatible longsustained release oil-core polyelectrolyte nanocarriers: from controlling physical state and stability to biological impact. Advances in Colloid and Interface Science, 222, 678-691.

Tang, S. Y., Manickam, S., Wei, T. K., \& Nashiru, B. (2012). Formulation development and optimization of a novel Cremophore EL-based nanoemulsion using ultrasound cavitation. Ultrasonics Sonochemistry, 19(2), 330-345.

Tegge, G. (1989). Yalpani, M.: Polysaccharides - SYNTHESIS, MODIFICATIONS AND STRUCTURE/PROPERTY RELATIONS (Vol. 36 of the series "Studies in Organic Chemistry"). Elsevier Science Publishers, Amsterdam - Oxford New York - Tokyo 1988. ISBN 0-444-43022-9. 522 pages, with over 40 tables and 130 schemes and illustrations. Price US \$ 171,-; Dfl 325,-. Available from : P.O. Box 211, 1000 AE Amsterdam (The Netherlands) or P.O. Box 1663, Grand Central Station. New York, NY 10163 (U.S.A.). Starch - Stärke, 41(6), 244-244.

Tomren, M. A., Másson, M., Loftsson, T., \& Tønnesen, H. H. (2007). Studies on curcumin and curcuminoids: XXXI. Symmetric and asymmetric curcuminoids: Stability, activity and complexation with cyclodextrin. International Journal of Pharmaceutics, 338(1-2), $27-34$.

Troncoso, E., Aguilera, J. M., \& McClements, D. J. (2012). Influence of particle size on the in vitro digestibility of protein-coated lipid nanoparticles. Journal of Colloid and Interface Science, 382(1), 110 116.

Vachoud, L., Zydowicz, N., \& Domard, A. (2000). Physicochemical behaviour of chitin gels. Carbohydrate Research, 326(4), 295-304.

Viana, R. B., da Silva, A. B. F., \& Pimentel, A. S. (2012). Infrared spectroscopy of anionic, cationic, and zwitterionic surfactants. Advances in Physical Chemistry, 2012, 14. 
Vlachos, N., Skopelitis, Y., Psaroudaki, M., Konstantinidou, V., Chatzilazarou, A., \& Tegou, E. (2006). Applications of Fourier transform-infrared spectroscopy to edible oils. Analytica Chimica Acta, 573-574, 459-465.

Yang, H., Irudayaraj, J., \& Paradkar, M. M. (2005). Discriminant analysis of edible oils and fats by FTIR, FT-NIR and FT-Raman spectroscopy. Food Chemistry, 93(1), 25-32.

Yu, H., Shi, K., Liu, D., \& Huang, Q. (2012). Development of a foodgrade organogel with high bioaccessibility and loading of curcuminoids. Food Chemistry, 131(1), 48-54.

Yucel, C., Quagliariello, V., Iaffaioli, R. V., Ferrari, G., \& Donsì, F. (2015). Submicron complex lipid carriers for curcumin delivery to intestinal epithelial cells: Effect of different emulsifiers on bioaccessibility and cell uptake. International Journal of Pharmaceutics, 494(1), 357-369.

Zhao, L., Du, J., Duan, Y., Zang, Y., Zhang, H., Yang, C., Cao, F., \& Zhai, G. (2012). Curcumin loaded mixed micelles composed of Pluronic P123 and F68: Preparation, optimization and in vitro characterization. Colloids and Surfaces B: Biointerfaces, 97, 101-108.

Publisher's Note Springer Nature remains neutral with regard to jurisdictional claims in published maps and institutional affiliations. 